\title{
Ultranonlocality and accurate band gaps from a meta-generalized gradient approximation
}

\author{
Thilo Aschebrock $\odot$ and Stephan Kümmel ${ }^{*}$ \\ Theoretical Physics IV, University of Bayreuth, 95440 Bayreuth, Germany
}

(Received 12 April 2019; revised manuscript received 1 July 2019; published 6 November 2019)

\begin{abstract}
The proper description of step structures in the exchange correlation potential, of charge localization, and a reasonable prediction of band gaps have been long-standing, serious challenges for semilocal density functionals. In practice, obtaining all of these properties from the functional derivative of an energy functional was possible only at the price of incorporating exact exchange. We here show that they can be achieved at significantly lower, semilocal computational expense by using kinetic-energy density-dependent functionals. The key to obtaining these features is a functional construction strategy that focuses on the derivative discontinuity and the density response.
\end{abstract}

DOI: 10.1103/PhysRevResearch.1.033082

\section{INTRODUCTION}

The success of density functional theory (DFT) is based on its favorable ratio of accuracy to computational cost. Semilocal functionals such as generalized gradient approximations (GGAs) [1-5] are to this day popular in solid-state physics and material science because of their moderate computational cost. They also continue to play a role, especially for largescale applications, in the molecular sciences, where hybrid functionals [6] have become the standard functionals for systems of small to moderate size. However, DFT based on semilocal functionals has also faced notorious problems, with prime examples known since the early days of DFT. In solid-state physics, the prediction of the fundamental (band) gap is an intrinsic limitation of explicit density-dependent semilocal functionals [7,8]. In material science, the proper description of charge localization and charge distributions between subsystems is a major challenge $[9,10]$ that affects many problems, e.g., surface adsorption $[11,12]$. In molecular physics and chemistry, missing field-counteracting terms have serious, detrimental consequences for the calculation of polarizabilities, hyperpolarizabilities, and charge transfer [13-15]. These problems originate from several fundamental shortcomings that are closely interconnected: a missing derivative discontinuity [16-18], a lack of step structures in the exchange correlation (xc) potential [19-22], and the delocalization error [23,24]. Density-driven errors [25,26] characterize many of these problems.

The standard approach to overcome the limitations is turning to functionals that are nonlocal. Self-interaction corrections can restore nonlocality [27-31], but the dominant

\footnotetext{
*Corresponding author: stephan.kuemmel@uni-bayreuth.de; http://www.tp4.uni-bayreuth.de/en

Published by the American Physical Society under the terms of the Creative Commons Attribution 4.0 International license. Further distribution of this work must maintain attribution to the author(s) and the published article's title, journal citation, and DOI.
}

and highly successful strategy of incorporating nonlocality has been the use of exact (Fock) exchange. By exploiting the adiabatic connection, yet higher accuracy can be reached [32], with DFT starting to rival wave-function-based methods in accuracy. However, the accuracy comes at a steep increase in computational cost. A standard hybrid functional is computationally already much more demanding than a GGA. Therefore, calculations incorporating Fock exchange are restricted to much smaller systems than semilocal calculations. This restriction can have serious consequences because often the interesting features of real-world systems, especially in the realm of nanomolecular and supramolecular science, stem from an intrinsic complexity that requires the explicit treatment of a large number of particles. Understanding light-harvesting systems [33-35] is a paradigm example: it requires calculating energy and charge transfer through arrays of dozens of chromophores, where each chromophore typically has hundreds of electrons. The chromophores in turn are typically embedded in a protein matrix, at least parts of which should also be taken into account explicitly [33,35-37]. However, semilocal functionals are currently at their limits for such systems due to their inability to properly describe charge transfer [38-40]. Therefore, there is a serious need for functionals that do not suffer from the large, qualitative errors that traditional semilocal functionals plague, yet come at a comparable computational price.

Here we demonstrate that one can achieve this with functionals that depend on the noninteracting kinetic energy density

$$
\tau(\mathbf{r})=\frac{\hbar^{2}}{2 m} \sum_{i=1}^{N}\left|\nabla \varphi_{i}(\mathbf{r})\right|^{2} .
$$

These so-called meta-GGAs are well established and their development has become an impressive success story [41-56]. They are the natural candidates for curing the abovementioned deficiencies because the explicit use of the (occupied) orbitals creates nonlocality since each $\varphi_{i}(\mathbf{r})$ depends on the density $n\left(\mathbf{r}^{\prime}\right)$ at all points $\mathbf{r}^{\prime}$. Yet, their computational cost is manifestly semilocal. In the following, we show how 
meta-GGA nonlocality can be led to unfold by focusing on the potential response and derivative discontinuity features.

Focusing the xc functional development less on the energy and also on the density or potential has already been advocated in principle [57-59] in the past, but in practice relied on expensive functionals $[58,60,61]$. The development of model potentials [62-66] has demonstrated that beneficial properties can be achieved semilocally. However, model potentials lack a corresponding energy functional and are not functional derivatives [63,67-69]. Furthermore, many of these constructions, as well as the attempt to restore the functional derivative property within the GGA form [70], lead to divergences [71-73]. Moreover, such purely multiplicative potentials are formally inappropriate to yield the fundamental gap [74].

The meta-GGA form allows to bring these earlier approaches to a unifying success. To this end, we write the exchange energy in the form

$$
E_{\mathrm{x}}^{\mathrm{mGGA}}[n]=A_{\mathrm{x}} \int n^{4 / 3} F_{\mathrm{x}}(s, \alpha) d^{3} r,
$$

where as usual $A_{\mathrm{x}}=-\left(3 e^{2} / 4\right)(3 / \pi)^{1 / 3}$, thereby satisfing the correct uniform coordinate density-scaling behavior [75]. Here the enhancement factor $F_{\mathrm{X}}(s, \alpha)$ is parametrized by the dimensionless variables

$$
s=\frac{|\nabla n|}{2\left(3 \pi^{2}\right)^{1 / 3} n^{4 / 3}}
$$

and $[51-54,76,77]$

$$
\alpha=\left(\tau-\tau^{\mathrm{W}}\right) / \tau^{\mathrm{unif}}
$$

where the von Weizsäcker kinetic energy density

$$
\tau^{W}=\frac{\hbar^{2}}{8 m} \frac{|\nabla n|^{2}}{n}
$$

is the single-orbital limit of $\tau$ and $\tau^{\text {unif }}=A_{\mathrm{s}} n^{5 / 3}$ with $A_{\mathrm{s}}=$ $\left(3 \hbar^{2} / 10 m\right)\left(3 \pi^{2}\right)^{2 / 3}$ its uniform-density limit. Spin polarization can be accounted for via the spin-scaling relation for exchange [78].

\section{ORIGIN OF ULTRANONLOCALITY}

In order to demonstrate that crucial (ultra)nonlocal features of exact exchange (EXX) can be captured by a meta-GGA, we recall that the EXX Kohn-Sham (KS) potential for a spinsaturated $N$-electron system

$$
\begin{aligned}
v_{\mathrm{x}}^{\mathrm{EXX}}(\mathbf{r})= & e^{2} \int \frac{n_{\mathrm{x}}^{\mathrm{EXX}}\left(\mathbf{r}, \mathbf{r}^{\prime}\right)}{\left|\mathbf{r}-\mathbf{r}^{\prime}\right|} d^{3} r^{\prime} \\
& +\frac{1}{n(\mathbf{r})} \sum_{i=1}^{N / 2}\left\{\left|\varphi_{i}(\mathbf{r})\right|^{2}\left(\bar{v}_{\mathrm{x} i}^{\mathrm{EXX}}-\bar{u}_{\mathrm{x} i}^{\mathrm{EXX}}\right)\right. \\
& \left.-\frac{\hbar^{2}}{m} \nabla \cdot\left[\psi_{i}^{*}(\mathbf{r}) \nabla \varphi_{i}(\mathbf{r})\right]\right\}+ \text { c.c. }
\end{aligned}
$$

can be decomposed [79] into the smooth Coulomb potential of the EXX hole $n_{\mathrm{x}}^{\mathrm{EXX}}$ (first term) and the response potential (second term). Here, a short overscore with index $i$ denotes taking the expectation value with the $i$ th orbital. Furthermore,

$$
u_{\mathrm{x} i}^{\mathrm{EXX}}(\mathbf{r})=\frac{1}{\varphi_{i}^{*}(\mathbf{r})} \frac{\delta E_{\mathrm{x}}^{\mathrm{EXX}}[n]}{\delta \varphi_{i}(\mathbf{r})}
$$

is the associated orbital-specific potential and $\psi_{i}(\mathbf{r})$ denotes the usual orbital shift of optimized effective potential (OEP) theory [80,81]. Hallmark nonlocal features of EXX result from the response potential $[13,15,79,82,83]$, in particular from the orbital-average terms on the right of Eq. (6). Note that the KS potential retains the very same mathematical structure [84] when the EXX energy is replaced by another orbital-dependent energy functional, e.g., a meta-GGA. The only difference is that EXX quantities are replaced by the corresponding meta-GGA quantities, e.g.,

$$
u_{\mathrm{x} i}^{\mathrm{mGGA}}(\mathbf{r})=\frac{1}{\varphi_{i}^{*}(\mathbf{r})} \frac{\delta E_{\mathrm{x}}^{\mathrm{mGGA}}[n]}{\delta \varphi_{i}(\mathbf{r})} .
$$

Thus, it is clear that, in principle, a meta-GGA can incorporate ultranonlocal features because the ultranonlocality of the potential stems from the orbital-average terms. For EXX, this nonlocality is enhanced by the Fock integrals. As meta-GGAs lack such integrals, one has to take special care to embed the nonlocality in the $\bar{v}_{\mathrm{x} i}^{\mathrm{mGGA}}-\bar{u}_{\mathrm{x} i}^{\mathrm{mGGA}}$ terms with proper magnitude and sign.

Guidance on how this can be achieved can be found by recalling that the ultranonlocality in the $\mathrm{KS}$ potential is closely connected $[15,85]$ to the derivative discontinuity

$$
\Delta_{\mathrm{x}}=\left.v_{\mathrm{x}}(\mathbf{r})\right|_{+}-\left.v_{\mathrm{x}}(\mathbf{r})\right|_{-}=\left.\frac{\delta E_{\mathrm{x}}[n]}{\delta n(\mathbf{r})}\right|_{+}-\left.\frac{\delta E_{\mathrm{x}}[n]}{\delta n(\mathbf{r})}\right|_{-},
$$

where the positive and negative signs denote evaluation of the functional derivative approaching the integer particle number from above and below, respectively. All experience to date is in line with $\Delta_{\mathrm{x}} \geqslant 0[7,86,87]$. When evaluating the functional derivative of a meta-GGA,

$$
\begin{aligned}
\frac{\delta E_{\mathrm{x}}^{\mathrm{mGGA}}[n]}{\delta n(\mathbf{r})}= & \frac{\delta}{\delta n(\mathbf{r})} \int e_{\mathrm{x}}(n, \nabla n, \tau) d^{3} r^{\prime} \\
= & \frac{\partial e_{\mathrm{x}}}{\partial n}(\mathbf{r})-\nabla \cdot\left[\frac{\partial e_{\mathrm{x}}}{\partial \nabla n}(\mathbf{r})\right] \\
& +\int \frac{\partial e_{\mathrm{x}}}{\partial \tau}\left(\mathbf{r}^{\prime}\right) \frac{\delta \tau\left(\mathbf{r}^{\prime}\right)}{\delta n(\mathbf{r})} d^{3} r^{\prime},
\end{aligned}
$$

only the $\tau$ dependence contributes to the derivative discontinuity as the first two terms correspond to the ones found in a usual GGA potential and originate from the explicit density dependence [85]. Therefore, and since $n(\mathbf{r})=\left.n(\mathbf{r})\right|_{-}=$ $\left.n(\mathbf{r})\right|_{+}$as well as $\tau(\mathbf{r})=\left.\tau(\mathbf{r})\right|_{-}=\left.\tau(\mathbf{r})\right|_{+}$,

$$
\Delta_{\mathrm{x}}^{\mathrm{mGGA}}=\int \frac{\partial e_{\mathrm{x}}}{\partial \tau}\left(\mathbf{r}^{\prime}\right)\left[\left.\frac{\delta \tau\left(\mathbf{r}^{\prime}\right)}{\delta n(\mathbf{r})}\right|_{+}-\left.\frac{\delta \tau\left(\mathbf{r}^{\prime}\right)}{\delta n(\mathbf{r})}\right|_{-}\right] d^{3} r^{\prime} .
$$

In order to simplify this into a transparent expression, we assume (similar to previous work [88]) that the first factor can be approximated by its average over the energetically relevant region of space. Thus,

$$
\Delta_{\mathrm{x}}^{\mathrm{mGGA}} \approx \overline{\frac{\partial e_{\mathrm{x}}}{\partial \tau}}\left[\left.\frac{\delta T_{\mathrm{s}}[n]}{\delta n(\mathbf{r})}\right|_{+}-\left.\frac{\delta T_{\mathrm{s}}[n]}{\delta n(\mathbf{r})}\right|_{-}\right]=\overline{\frac{\partial e_{\mathrm{x}}}{\partial \tau}} \Delta_{s},
$$


where $T_{\mathrm{s}}[n]$ is the usual noninteracting kinetic energy functional and $\Delta_{s}$ the corresponding non-negative KS gap. Equation (12) thus establishes a link between the magnitude and sign of $\partial e_{\mathrm{x}} / \partial \tau$ and $\Delta_{\mathrm{x}}^{\mathrm{mGGA}} .1$

A meta-GGA with a sizable discontinuity of proper sign can therefore be constructed by requesting

$$
\partial e_{\mathrm{x}} / \partial \tau>0,
$$

as this ensures

$$
\overline{\partial e_{\mathrm{x}} / \partial \tau}>0
$$

for any system. For a parametrization in $s$ and $\alpha$ [cf. Eq. (2)],

$$
\frac{\partial e_{\mathrm{x}}}{\partial \tau}=\frac{A_{\mathrm{x}}}{A_{\mathrm{s}}} \frac{1}{n^{1 / 3}} \frac{\partial F_{\mathrm{x}}}{\partial \alpha} .
$$

With $A_{\mathrm{s}}$ and $n$ positive and $A_{\mathrm{x}}<0$, a positive $\Delta_{\mathrm{x}}^{\mathrm{mGGA}}$ follows from

$$
\partial F_{\mathrm{x}} / \partial \alpha<0
$$

and a larger negative slope in the energetically relevant regions of space can be expected to lead to a larger $\Delta_{\mathrm{x}}^{\mathrm{mGGA}}$. We further note that the sign in Eq. (16) goes well along with the conjectured strongly tightened bound

$$
F_{\mathrm{x}}(s, \alpha) \leqslant F_{\mathrm{x}}(s, \alpha=0) \leqslant 1.174
$$

for all $\alpha$ [89]. It is also in line with the observations of ultranonlocality for the polarization dependence of solids [90] in the meta-GGA kernel [88] (see the detailed discussion in Appendix C). Furthermore, as $\alpha$ is a well-established measure of electron localization [51,91,92], Eq. (16) can be interpreted as a condition to energetically favor electron localization via the exchange energy, as EXX does.

\section{PROOF OF CONCEPT}

In order to demonstrate how these findings can be used in actual meta-GGA construction, we proceed in two steps. First, as a proof of concept $(\mathrm{PoC})$ we discuss the simple, conceptual enhancement factor

$$
F_{\mathrm{x}}^{\mathrm{PoC}}(\alpha)=c_{\mathrm{H}}-c_{1} \alpha /\left(1+c_{2} \alpha\right)
$$

which uses only $\alpha$ and three parameters. This will not yield a generally useful functional, but allows to clearly demonstrate the connection between Eq. (16) and practically important manifestations of meta-GGA (ultra)nonlocality. We chose

$$
c_{\mathrm{H}}=\frac{40}{81}\left(\frac{4 \pi^{2}}{3}\right)^{1 / 3}
$$

to obtain the correct hydrogen atom energy (cf. Appendix B for a derivation). The parameters $c_{1}$ and $c_{2}$ determine the slope and the curvature of $F_{\mathrm{x}}^{\mathrm{PoC}}(\alpha)$ and will be discussed in the following.

\footnotetext{
${ }^{1}$ Note that the above arguments do not exclude the possibility that $\Delta_{\mathrm{x}}^{\mathrm{mGGA}} \neq 0$ for $\Delta_{s}=0$; the former is in principle possible when ones go beyond the averaging approximation of Eq. (12). Further note that the argument formulated here for $\Delta_{\mathrm{x}}$ can readily be extended to $\Delta_{\mathrm{xc}}$.
}

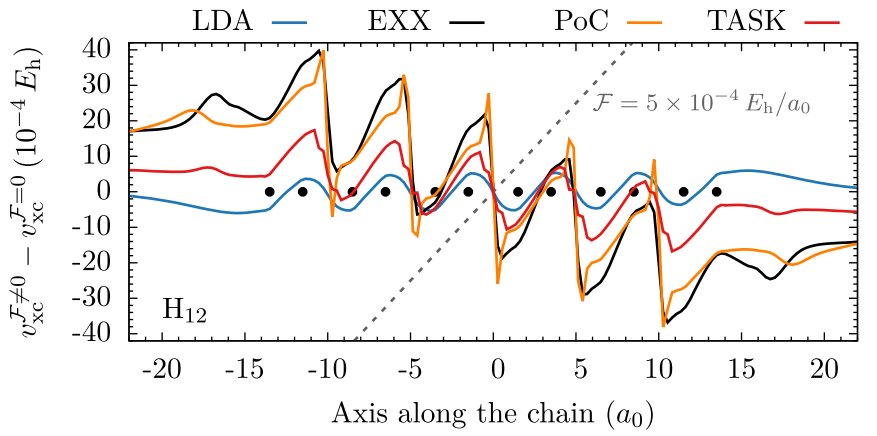

FIG. 1. Difference between the $\mathrm{x}(\mathrm{c})$ potentials (OEP) with and without a homogeneous external electric field for a chain of 12 hydrogen atoms at alternating distances of $2 a_{0}$ and $3 a_{0}$ (cf. Appendix D for computational details).

As a challenging, paradigm test of nonlocality we analyze the response potential of hydrogen chains [13,30,97-103]. Figure 1 shows the potential response obtained from LDA and EXX for a $\mathrm{H}_{12}$ chain in a static electric field, demonstrating the well-known field-counteracting term of EXX [13] and its absence in LDA. The striking observation in Fig. 1 is that $F_{\mathrm{x}}^{\mathrm{POC}}$ can achieve a potential response that is very close to the one of EXX with a suitable choice of parameters, namely, $c_{1}=27 / 40$ and $c_{2}=c_{1} /\left(3+c_{\mathrm{H}}\right)$. Table I shows that the polarizabilities for hydrogen chains obtained with this $F_{\mathrm{x}}^{\mathrm{PoC}}$ are close to the coupled-cluster (CCSDT) reference values for all chain lengths. Hence, this meta-GGA exhibits the correct trend with increasing chain length that so far has not been accessible with any semilocal functional due to the lack of the field-counteracting term. This is a proof of concept that even a simple meta-GGA can yield strong ultranonlocality.

Furthermore, this simple meta-GGA allows to transparently and explicitly demonstrate the relation between the derivative $\partial F_{\mathrm{x}} / \partial \alpha$ and the field-counteracting term in the KS potential. By choosing different values for the parameters $c_{1}$ and $c_{2}$, different slopes in $\alpha$ can easily be realized. For a first alternative (1st alt.) PoC meta-GGA we opt for the opposite slope at $\alpha=0$ compared to the original (orig.) $\mathrm{PoC}$

TABLE I. Polarizability calculated from the dipole moment in $a_{0}{ }^{-3}$ for hydrogen chains of different length and x(c) energy functionals. CCSD(T) values from Ref. [94]. DFT calculations were performed self-consistently in a locally modified version of PARSEC [95,96], orbital-dependent functionals treated in full OEP unless noted otherwise (cf. Appendix D).

\begin{tabular}{lccrcc}
\hline \hline & $\mathrm{H}_{4}$ & $\mathrm{H}_{6}$ & \multicolumn{1}{c}{$\mathrm{H}_{8}$} & $\mathrm{H}_{12}$ & $\mathrm{H}_{18}$ \\
\hline LDA (xc) [93] & 37.6 & 72.8 & 115.0 & 211.4 & 369 \\
PKZB (xc) [45] & 35.5 & 65.8 & 105.1 & 189.1 & 323 \\
$\tau$-HCTH (xc) [46] & 35.7 & 68.6 & 96.9 & 194.3 & 335 \\
MVS (x) [54] & 34.0 & 63.6 & 102.3 & 181.5 & 308 \\
SCAN (xc) [52] & 35.4 & 67.8 & 106.6 & 192.3 & 329 \\
TASK (x) & 34.0 & 62.9 & 96.2 & 169.8 & 286 \\
PoC (x) & 30.3 & 51.6 & 74.4 & 121.7 & 194 \\
EXX (x) & 32.1 & 56.5 & 83.0 & 138.7 & 225 \\
CCSD(T) & 28.8 & 50.5 & 74.1 & 123.6 & 200 \\
\hline \hline
\end{tabular}




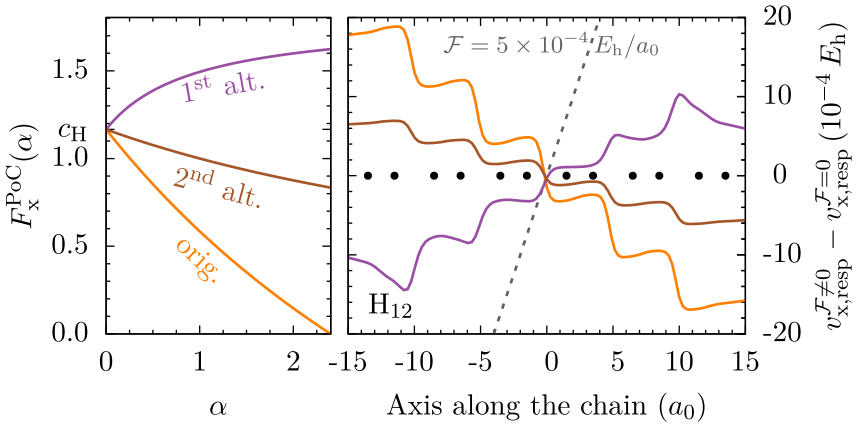

FIG. 2. Explicit demonstration of the relation between $\partial F_{\mathrm{x}} / \partial \alpha$ and the field-counteracting term in $v_{\mathrm{x}}(\mathbf{r})$. Left: original and alternative PoC enhancement factors. Right: difference between the exchange response potentials (KLI) with and without a homogeneous external electric field of strength $\mathcal{F}=5 \times 10^{-4} E_{h} / a_{0}$ for the $\mathrm{H}_{12}$ chain evaluated with the respective enhancement factors.

and use the Lieb-Oxford bound [104] as a natural limiting value for $\alpha \rightarrow \infty$ and thus as the upper bound. This leads to $c_{1}=-27 / 40$ and $c_{2}=-c_{1} /\left(1.804-c_{\mathrm{H}}\right)$. The left panel of Fig. 2 shows the resulting 1 st alt. PoC enhancement factor as a function of $\alpha$. It has a strictly positive slope, contrary to the strictly negative one of the orig. PoC meta-GGA. The right panel of Fig. 2 displays the difference between the exchange response potential [in Krieger-Li-Iafrate (KLI) approximation [82]] [cf. decomposition in hole and response potential as in Eq. (6), with and without a homogeneous external electric field for the $\mathrm{H}_{12}$ chain]. While the orig. PoC meta-GGA exhibits a pronounced field-counteracting behavior, the 1 st alt. PoC meta-GGA also displays substantial ultranonlocality in the potential, but with the opposite sign and thus unphysically enhances the external field. This clearly demonstrates the link between $\partial F_{\mathrm{x}} / \partial \alpha<0$ and the field-counteracting term in the KS potential. In similar fashion one can transparently alter the slope $\partial F_{\mathrm{x}} / \partial \alpha$ in the PoC meta-GGA form to show that the amplitude of $\partial F_{\mathrm{x}} / \partial \alpha$ is directly correlated to the amplitude of the field-counteracting term in the KS potential. To this end, we construct a second alternative (2nd alt.) PoC meta-GGA with $c_{1}=c_{\mathrm{H}}\left(c_{\mathrm{H}}-1\right)$ and $c_{2}=c_{\mathrm{H}}-1$, where these parameters are determined by the homogeneous electron gas limit, $F_{\mathrm{x}}(\alpha=1)=1$, and by the negativity of exchange energy density, i.e., $\lim _{\alpha \rightarrow \infty} F_{\mathrm{x}}(\alpha)=0$. Figure 2 shows that the restricted slope of $F_{\mathrm{X}}(\alpha)$ of this 2 nd alt. PoC meta-GGA also limits the amplitude of the field-counteracting term in the KS potential.

Another stringent test of discontinuity features that probes the delocalization problem is defined by the $\mathrm{H}_{8}-\mathrm{H}_{8}$ system of Ref. [9]. To the best of our knowledge, no semilocal functional to date has passed this test and even regular and local hybrids fail it $[9,105]$. Two aligned, but well-separated hydrogen chains are exposed to a constant external electric field of increasing strength in a series of ground-state calculations. As the field strength increases, charge eventually transfers from one chain to the other. Due to their large separation of $8 \AA$, integer preference [106] must be preserved, i.e., charge transfer should only occur via integer electron jumps at specific field strengths. However, as shown in the upper panel of Fig. 3, the local density approximation (LDA) leads to a gradual transfer of fractional charges, and usual semilocal

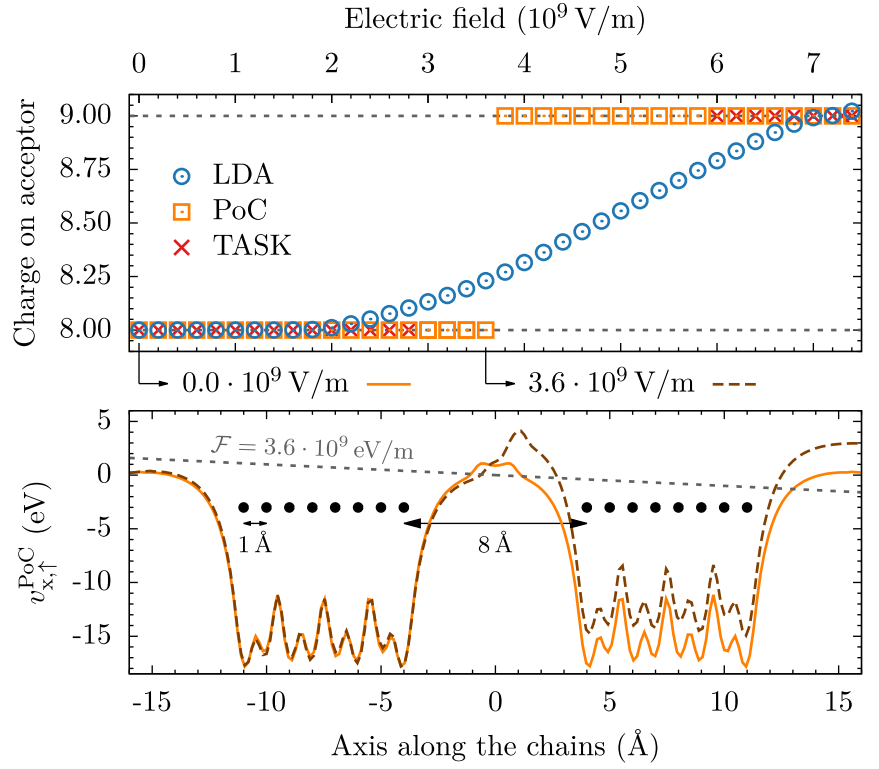

FIG. 3. Top: integrated (unsigned) charge on the $\mathrm{H}_{8}$ acceptor as a function of the external field for LDA, as well as the PoC and TASK meta-GGAs. Bottom: plots of the exchange potential revealing the step structure in the PoC meta-GGA, by which the principle of integer preference is enforced. See Appendix E for computational details.

functionals and hybrids show similar behavior [9]. The upper panel of Fig. 3 shows that $F_{\mathrm{x}}^{\mathrm{PoC}}$ (with the same choice of parameters that lead to good hydrogen chain polarizabilities) also correctly restores integer preference. If a step function would be used as an external field and the chains would be infinitely far apart, the critical step size associated with an integer electron transfer would be given directly [17] by the fundamental gap

$$
I-A=\Delta_{\mathrm{s}}+\Delta_{\mathrm{xc}}
$$

of a single $\mathrm{H}_{8}$ chain. The situation considered here is more complex due to the finite distance as well as the linear field that induces a considerable polarization of both chains. Nevertheless, the $\Delta_{\mathrm{xc}}$ contribution remains pivotal for a correct description of this charge transfer within KS theory. It manifests itself in the KS potential [21] as depicted in the lower panel of Fig. 3, which demonstrates that $F_{\mathrm{x}}^{\mathrm{PoC}}$ leads to the right properties for the right reason: At $\mathcal{F}=3.6 \times 10^{9} \mathrm{eV} / \mathrm{m}$, which would lead to a fractional charge transfer for any conventional semilocal functional, a pronounced step structure that counteracts the charge transfer is observed. Similarly, after the jump a potential step with opposite sign stabilizes the charge transfer. This is exactly the behavior that EXX exhibits and by which a nonlocal self-interaction correction cures the charge-transfer problem [21].

\section{TASK: A GENERAL PURPOSE META-GGA}

After this proof of concept as to how nonlocality can be achieved in meta-GGA construction, we take a second step and devise a more generally useful meta-GGA for exchange that features nonlocality. The outer framework of this functional, named TASK for the initials of the authors, is adopted 
from the exchange part of the SCAN meta-GGA [52] and reads as

$$
F_{\mathrm{x}}^{\mathrm{TASK}}(s, \alpha)=h_{\mathrm{x}}^{0} g_{\mathrm{x}}(s)+\left[1-f_{\mathrm{x}}(\alpha)\right]\left[h_{\mathrm{x}}^{1}(s)-h_{\mathrm{x}}^{0}\right]\left[g_{\mathrm{x}}(s)\right]^{d} .
$$

We likewise choose

$$
F_{\mathrm{x}} \leqslant h_{x}^{0}=1.174
$$

as an upper bound, thereby obeying the rigorous strongly tightened bound [89] for $\alpha=0$, as well as the more strict conjectured bound given by Eq. (17). By implying $f_{\mathrm{x}}(\alpha=$ $0)=1$ this meta-GGA is identical to SCAN for one-orbital systems $(\alpha=0)$, with

$$
g_{\mathrm{x}}(s)=1-\exp \left(-c s^{-1 / 2}\right),
$$

where $c=4.9479$ was obtained to recover the exact hydrogen atom energy. Moreover, $g_{\mathrm{x}}(s)$ governs $F_{\mathrm{x}}(s, \alpha)$ for $s \rightarrow \infty$ and vanishes like $s^{-1 / 2}$ [89], making the exchange energy per particle scale correctly to a negative constant under nonuniform coordinate scaling to the true two-dimensional limit $[107,108]$.

We chose $h_{\mathrm{x}}^{1}(s)$ to only depend on $s$. In this way, we can readily satisfy the construction principle of a negative slope in $\alpha$ of sizable magnitude [cf. Eq. (16)] to preserve the desired field-counteracting properties. Furthermore, we determine $f_{\mathrm{x}}(\alpha)$ and $h_{\mathrm{x}}^{1}(s)$ by recovering the fourth-order gradient expansion (GE4) for exchange [109], valid for slowly varying densities with small $s$ and $\alpha \approx 1$. We do this in a way, however, that deviates decisively from SCAN, where the GE4 is solely recovered by $h_{\mathrm{x}}^{1}$ and without a leading-order contribution of $\alpha$, as $f_{\mathrm{x}}(\alpha)$ is chosen to be flat, i.e., to vanish in any power of $\nabla n$. Our aim, however, is to fulfill Eq. (16) with an appreciable slope, and therefore we require nonvanishing GE4 contributions of $f_{\mathrm{x}}(\alpha)$ and thus $\alpha$ to all relevant orders. To this end, we start from the general expression for the gradient expansion around $s=0$ and $\alpha=1$,

$$
\begin{aligned}
F_{\mathrm{x}}^{\mathrm{GE} 4}(s, \alpha) \sim & 1+\mu_{s} s^{2}+\mu_{\alpha}(\alpha-1)+C_{s} s^{4}+C_{s \alpha} s^{2}(\alpha-1) \\
& +C_{\alpha}(\alpha-1)^{2}+\mathcal{O}\left(\nabla^{6}\right),
\end{aligned}
$$

and then make use of the GE4 of $\tau[110,111]$ as detailed in Appendix A. Comparison with the usual GE4 for exchange then leads to the coefficients

$$
\begin{aligned}
& \mu_{s}=\frac{10+60 \mu_{\alpha}}{81}, \quad C_{s}=-\frac{1606-50 \mu_{\alpha}}{18225}, \\
& C_{s \alpha}=-\frac{511-50 \mu_{\alpha}}{13500}, \quad C_{\alpha}=\frac{73-50 \mu_{\alpha}}{5000}
\end{aligned}
$$

and one degree of freedom, the choice of $\mu_{\alpha}$. However, due to the separation of variables with respect to $s$ and $\alpha$ in our ansatz for the enhancement factor of Eq. (21), the mixed fourth-order term in the GE4 of Eq. (24) is generated by multiplication and is thereby not independent of the secondorder terms. Therefore, in our construction, the corresponding mixed coefficient $C_{s \alpha}$ is directly linked to the second-order coefficients via

$$
\mu_{s} \mu_{\alpha}=\left(1-h_{\mathrm{x}}^{0}\right) C_{s \alpha},
$$

which implies two possible values for $\mu_{\alpha}$ :

$$
\mu_{\alpha}^{ \pm}=-\frac{97+3 h_{\mathrm{x}}^{0} \pm \sqrt{9\left(h_{\mathrm{x}}^{0}\right)^{2}+74166 h_{\mathrm{x}}^{0}-64175}}{1200} .
$$

To obtain a sizable derivative discontinuity we choose the more negative solution

$$
\mu_{\alpha}=\mu_{\alpha}^{+} \approx-0.209897,
$$

and, thus, a pronounced negative slope in $\alpha$ in accordance with Eq. (16).

To satisfy the listed constraints while obtaining a smooth $F_{\mathrm{x}}(s, \alpha)$, we choose the following ansatz:

$$
h_{\mathrm{x}}^{1}(s)=\sum_{\nu=0}^{2} a_{\nu} R_{\nu}\left(s^{2}\right), \quad f_{\mathrm{x}}(\alpha)=\sum_{\nu=0}^{4} b_{\nu} R_{\nu}(\alpha),
$$

which is based on Chebyshev rational functions [112,113] $R_{v}(x)$ of degree $v$. The eight coefficients are determined by

$$
h_{\mathrm{x}}^{1}(0)=1, \quad f_{\mathrm{x}}(0)=1, \quad f_{\mathrm{x}}(1)=0
$$

as well as the values of

$$
\left.\frac{\partial^{2} h_{\mathrm{x}}^{1}}{\partial s^{2}}\right|_{s=0},\left.\quad \frac{\partial^{4} h_{\mathrm{x}}^{1}}{\partial s^{4}}\right|_{s=0},\left.\quad \frac{\partial f_{\mathrm{x}}}{\partial \alpha}\right|_{\alpha=1},\left.\quad \frac{\partial^{2} f_{\mathrm{x}}}{\partial \alpha^{2}}\right|_{\alpha=1}
$$

that follow from imposing the GE4 as given by Eq. (24). The final condition to determine the coefficients can be formulated as choosing the limiting value of $f_{\mathrm{x}}(\alpha)$ as $\alpha \rightarrow \infty$. In order to ensure $\partial F_{\mathrm{x}} / \partial \alpha<0$ everywhere as well as negativity of the exchange energy density, $f_{\mathrm{x}}(\alpha \rightarrow \infty)$ has to be limited to the interval ] $-4.4,-2.5$ [ approximately. We here choose

$$
f_{\mathrm{x}}(\alpha \rightarrow \infty)=-3
$$

as a good balance between a pronounced negative slope for small values of $\alpha$ and minimizing undesired curvature in $\alpha$. We find $a_{0}=0.938719, a_{1}=-0.076371$, $a_{2}=-0.0150899, b_{0}=-0.628591, b_{1}=-2.10315, b_{2}=$ $-0.5, b_{3}=0.103153, b_{4}=0.128591$.

Remarkably, the central premise of Eq. (16) follows naturally from the ansatz (21) when one opts for recovering the GE4 with a nonvanishing leading-order $\alpha$ contribution. Finally, we choose $d=10$ to bundle $F(s, \alpha)$ quickly for $s \rightarrow \infty$, thereby restricting numerical issues that may arise as $\mathbf{r} \rightarrow \infty$ in the asymptotic region of finite systems, as well as to enforce negativity of the exchange energy density. In summary, our TASK functional fulfills all of the exact constraints for exchange that were imposed on SCAN (yet only the hydrogen atom norm), and in addition achieves a more pronounced negative slope in Eq. (16) that is crucial for field-counteracting properties. The resulting enhancement factor of TASK is depicted in Fig. 4. The constraints force the TASK functional toward a field-counteracting term that is somewhat less pronounced than the one of $F_{\mathrm{x}}^{\mathrm{PoC}}$ and EXX, as seen in Fig. 1. However, as seen in Table I the polarizabilities are still substantially improved compared to LDA and SCAN. Also, the upper panel of Fig. 3 depicts a clear improvement over LDA for the $\mathrm{H}_{8}-\mathrm{H}_{8}$ charge-transfer system.

The potential that we obtain is relatively smooth and we did not experience difficulties in the numerical representation. By fulfilling Eq. (16), one guarantees that as functions of 


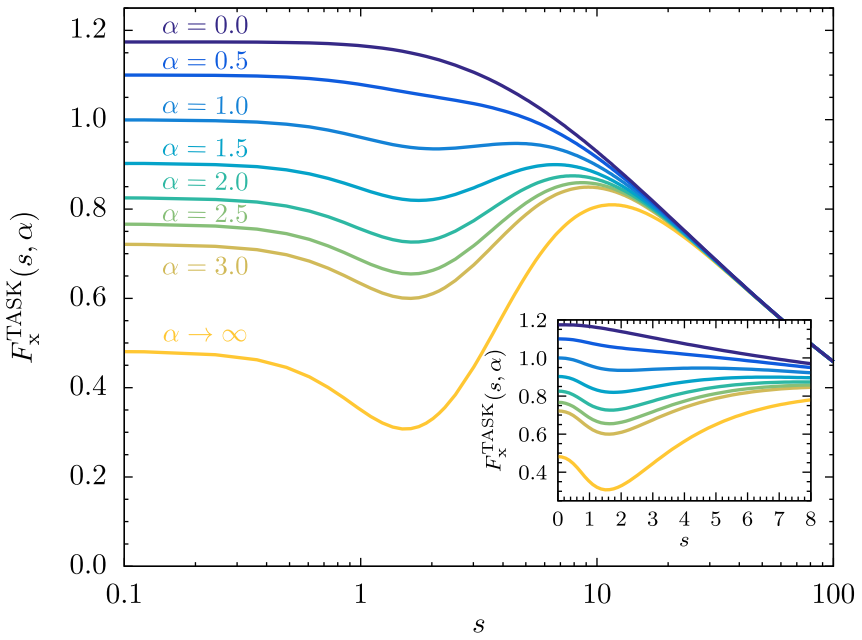

FIG. 4. TASK exchange enhancement factor $F_{\mathrm{x}}^{\mathrm{TASK}}(s, \alpha)$ as a function of $s$ for fixed values of $\alpha$.

$s$ curves for different $\alpha$ do not cross, a condition that has been associated with numerical stability [52]. Several metaGGAs that have previously been constructed have numerical features that require special attention in their computational evaluation, and the underlying reasons have been studied with a new iso-orbital indicator [114].

We here deliberately focused on the exchange functional because exchange is decisive for curing charge-transfer errors. A recent study [118] furthermore demonstrated that correlation contributes to the field-counteracting term in a similar fashion as exchange. Therefore, it is not unreasonable to cover the field-counteracting terms by meta-GGA exchange. Ground-state energetics, on the other hand, usually benefit from a correlation functional which is especially tailored toward the corresponding semilocal exchange. However, constructing such a correlation functional is a task in itself, to be addressed in future work. Therefore, we presently combine the TASK exchange with PW92-LDA correlation [93], as LDA is universal and not tied to a specific exchange functional.

We calculated atomization energies for a set of diatomic molecules. The set is small, but comprises single, double, and triple bonds and thus gives a first impression of binding properties. The mean absolute error for the atomization energy are reported in Table II. Without further context, TASK+ PW92 accuracy for atomization energies is not impressive. However, one can here see a parallel between meta-GGAs and hybrid functionals: Hybrids with a small (ca. 20\%) fraction of Fock exchange are good for atomization energies. A reliable description of charge transfer and charge localization, however, requires much larger (up to 75\%) fractions [125] and correspondingly leads to a considerably poorer description of atomization energies [126]. Therefore, we see it as a sign of hope that TASK+PW92 atomization energies are at least significantly better than LDA while at the same time successfully tackling the charge-transfer errors. Furthermore, as the TASK functional is constructed in purely nonempirical fashion, it can be expected to be reliable over a wide range of systems and situations.
TABLE II. Atomization energies in $\mathrm{kcal} / \mathrm{mol}$ of diatomic molecules based on self-consistent KS calculations at experimental bond lengths and mean absolute error (MAE) across the test set. The calculations were performed on a real-space prolate spheroidal grid with DARSEC [115], an all-electron code for single atoms or diatomic molecules. SCAN, EXX, and TASK (here in combination with PW92-LDA correlation) potentials were evaluated in the KLI approximation [82]. The experimental values (with zero point vibration removed) and the experimental bond lengths are taken from Ref. [116] (cf. the Supplemental Material of Ref. [117] for computational details).

\begin{tabular}{crrrrrr}
\hline \hline Molecule & EXX & LDA & PBE & SCAN & TASK & Expt. \\
\hline $\mathrm{H}_{2}$ & 83.8 & 112.9 & 104.6 & 107.7 & 117.0 & 109.5 \\
$\mathrm{LiH}$ & 34.1 & 60.8 & 53.5 & 55.6 & 58.9 & 58.0 \\
$\mathrm{Li}_{2}$ & 3.6 & 23.8 & 19.9 & 18.1 & 11.6 & 24.7 \\
$\mathrm{LiF}$ & 90.5 & 156.3 & 139.0 & 138.1 & 130.2 & 138.3 \\
$\mathrm{CO}$ & 172.3 & 299.2 & 269.1 & 255.2 & 248.2 & 259.5 \\
$\mathrm{~N}_{2}$ & 112.4 & 268.0 & 243.9 & 220.9 & 174.9 & 228.5 \\
$\mathrm{NO}$ & 47.6 & 199.4 & 170.9 & 146.5 & 122.9 & 152.5 \\
$\mathrm{OH}$ & 65.4 & 123.2 & 105.0 & 94.9 & 97.6 & 106.4 \\
$\mathrm{O}_{2}$ & 30.0 & 175.1 & 144.1 & 126.8 & 131.0 & 120.5 \\
$\mathrm{FH}$ & 96.6 & 162.0 & 142.0 & 140.4 & 139.2 & 141.1 \\
$\mathrm{~F}_{2}$ & -41.7 & 78.0 & 52.8 & 37.4 & 23.4 & 38.4 \\
$\mathrm{MAE}$ & 62.1 & 25.7 & 9.1 & 4.6 & 14.6 & \\
\hline \hline
\end{tabular}

Further evidence that the TASK functional indeed incorporates correct physics and yields a sizable $\Delta_{\mathrm{x}}^{\mathrm{mGGA}}$ is seen when calculating band gaps. For the study of gaps, we resorted to general KS (gKS) theory [127], as it gives direct access to the

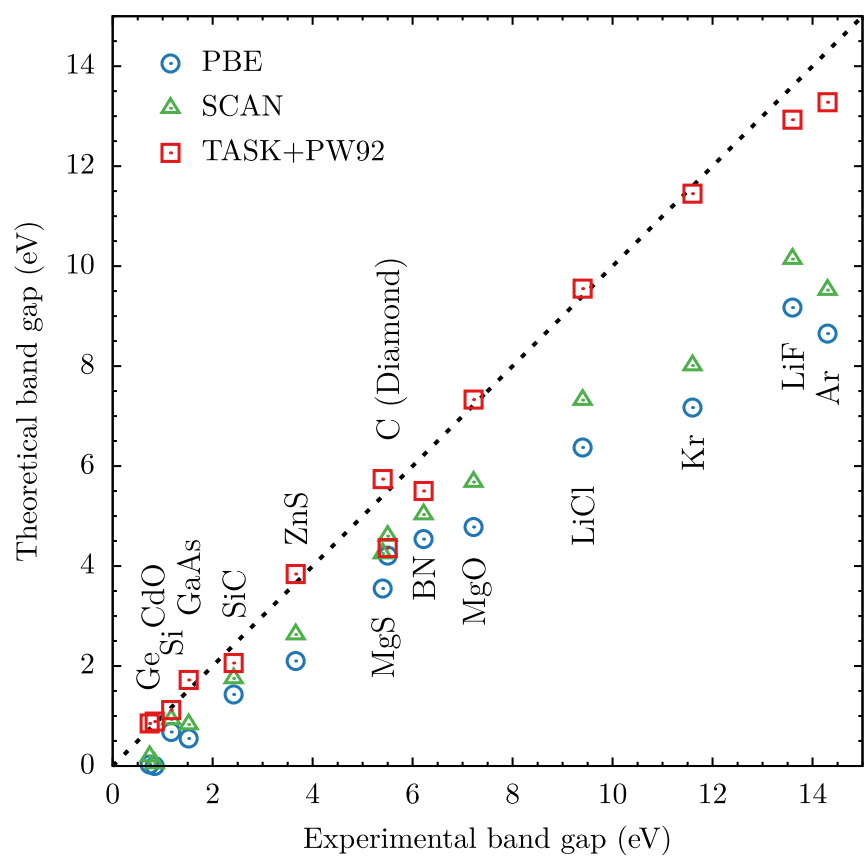

FIG. 5. Experimental band gaps compared to band gaps computed by PBE [4], SCAN (gKS) [52], and TASK+ PW92 (gKS). See Table III for values and further details. 
TABLE III. Structure, experimental geometrical parameters, and fundamental band gaps of the solids considered in this work. All band gaps are obtained from band structures that are sampled with a step size of $0.02 a_{0}{ }^{-1}$.

\begin{tabular}{|c|c|c|c|c|c|c|}
\hline \multirow[b]{2}{*}{ Solid } & \multirow[b]{2}{*}{ Structure } & \multirow[b]{2}{*}{ Lattice parameter $(\AA)$} & \multicolumn{4}{|c|}{ Fundamental band gaps $(\mathrm{eV})$} \\
\hline & & & PBE & SCAN & TASK+PW92 & Expt. \\
\hline $\mathrm{Ge}$ & Diamond & 5.652 [119] & 0.03 & 0.19 & 0.85 & $0.74[120]$ \\
\hline $\mathrm{CdO}$ & Rock salt & $4.704 \quad[121]$ & 0.00 & 0.05 & 0.89 & $0.84[122]$ \\
\hline $\mathrm{Si}$ & Diamond & $5.430[119]$ & 0.68 & 0.93 & 1.12 & $1.17[120]$ \\
\hline GaAs & Zinc blende & $5.648[119]$ & 0.55 & 0.83 & 1.72 & $1.52[120]$ \\
\hline $\mathrm{SiC}$ & Zinc blende & 4.358 [119] & 1.43 & 1.75 & 2.06 & $2.42[120]$ \\
\hline $\mathrm{ZnS}$ & Zinc blende & $5.409[120]$ & 2.10 & 2.63 & 3.84 & $3.66[120]$ \\
\hline $\mathrm{MgS}$ & Zinc blende & $5.622[120]$ & 3.55 & 4.25 & 5.74 & $5.4[120]$ \\
\hline $\mathrm{C}$ & Diamond & $3.567 \quad[119]$ & 4.21 & 4.60 & 4.36 & $5.48[120]$ \\
\hline $\mathrm{BN}$ & Zinc blende & $3.603[120]$ & 4.54 & 5.03 & 5.50 & $6.22[120]$ \\
\hline $\mathrm{MgO}$ & Rock salt & 4.207 [119] & 4.78 & 5.68 & 7.33 & $7.22[120]$ \\
\hline $\mathrm{LiCl}$ & Rock salt & $5.106[119]$ & 6.37 & 7.32 & 9.55 & 9.4 [123] \\
\hline $\mathrm{Kr}$ & fcc & $6.130[123]$ & 7.17 & 8.01 & 11.45 & 11.6 [123] \\
\hline $\mathrm{LiF}$ & Rock salt & $4.010[123]$ & 9.17 & 10.14 & 12.93 & 13.6 [124] \\
\hline $\mathrm{Ar}$ & fcc & $5.310[123]$ & 8.65 & 9.52 & 13.28 & $14.3[124]$ \\
\hline
\end{tabular}

fundamental gap [74,124,128,129], i.e.,

$$
\Delta_{\mathrm{s}}^{\mathrm{gKS}} \approx \Delta_{\mathrm{s}}+\Delta_{\mathrm{xc}} .
$$

All calculations were performed at the experimental geometry with a modified version of the periodic all-electron code BAND [130], a $9 \times 9 \times 9$ Monkhorst-Pack k grid [131], and the TZ2P [132] basis set (except for $\mathrm{Ar}$ and $\mathrm{Kr}$, for which QZ4P is used). The scalar relativistic effects are included by the ZORAmethod [133]. Figure 5 shows that gaps calculated with TASK in combination with PW92-LDA correlation are uniformly and significantly improved compared to usual semilocal results, here represented by PBE [4], for systems ranging from traditional semiconductors to wide-gap insulators (see Table III for detailed numbers). For most systems studied, TASK also performs significantly better than SCAN, which was reported [124] to already yield considerably more

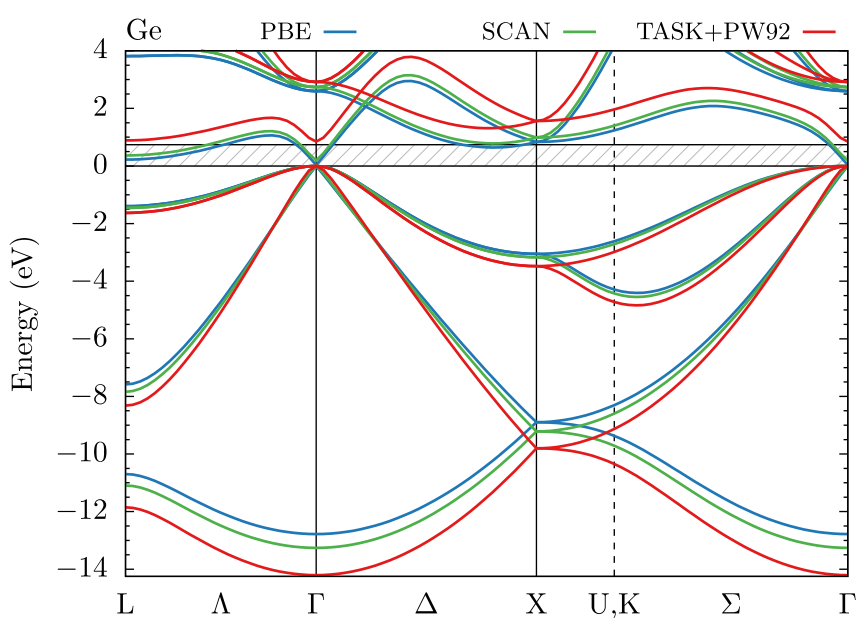

FIG. 6. Band structure of Ge calculated with PBE [4] (blue), SCAN [52] (green), and TASK+ PW92 (red) at the experimental geometry (meta-GGAs calculated in gKS scheme). The top of the valance band is the zero of the energy scale. The experimental fundamental band gap is visually indicated by the hatched region. realistic band gaps. It is noticeable that TASK band gaps are remarkably close to experiment for a wide range of materials, reaching from the semiconductors $\mathrm{Ge}, \mathrm{CdO}, \mathrm{Si}$, and $\mathrm{GaAs}$ to minerals such as $\mathrm{MgO}$ and $\mathrm{LiCl}$. The functional also gives significantly improved values for the large-gap insulators $\mathrm{Kr}$, LiF, and Ar. Most notably, TASK opens the band gap with a magnitude close to the experimental value for $\mathrm{Ge}$ and $\mathrm{CdO}$, whereas PBE and SCAN incorrectly predict both systems to be metallic (see Figs. 6 and 7 for the corresponding band structures).

\section{SUMMARY AND CONCLUSIONS}

In summary, we laid out an exchange-correlation functional construction strategy that takes into account properties of the KS potential such as the derivative discontinuity and

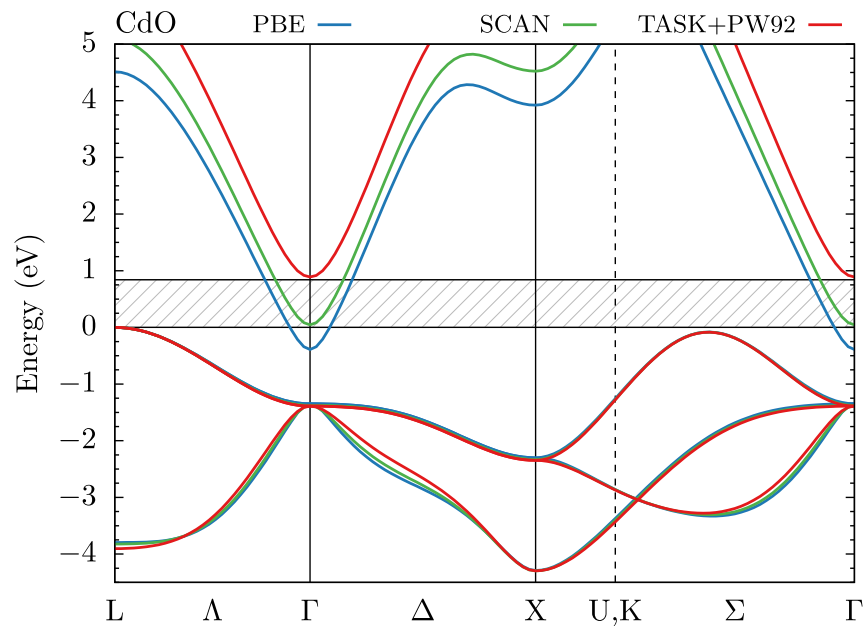

FIG. 7. Band structure of $\mathrm{CdO}$ calculated with PBE [4] (blue), SCAN [52] (green), and TASK+ PW92 (red) at the experimental geometry (meta-GGAs calculated in gKS scheme). The top of the valance band is the zero of the energy scale. The experimental fundamental band gap is visually indicated by the hatched region. 
the density response in addition to the ground-state energy. We derived a transparent general criterion for obtaining a sizable derivative discontinuity from a meta-GGA. In a proofof-concept construction we demonstrated that this criterion directly governs the response potential and the ultranonlocality of a meta-GGA. Taking into account known exact constraints, we further developed a nonempirical, general-purpose metaGGA for exchange that yields improved response properties and band gaps. This suggests that the ground-state energy need not be the only focus of meta-GGA construction, but construction strategies can also target the density and the potential. Our work shows that meta-GGAs can live up to the orbital functional promise of yielding ultranonlocal features without requiring costly exchange integrals. The meta-GGA form thus continues to hold great promise for future functional development.

\section{ACKNOWLEDGMENTS}

We acknowledge discussion with A. Kaiser and F. Hofmann. Financial support from the Elite Study Program
"Biological Physics" of the Elite Network of Bavaria, from the German-Israeli Foundation for Scientific Research and Development, from the Bavarian State Ministry of Science, Research, and the Arts for the Collaborative Research Network "Solar Technologies go Hybrid," and from the Bavarian Polymer Institute in terms of computing resources, is gratefully acknowledged.

\section{APPENDIX A: FOURTH-ORDER GRADIENT EXPANSION FOR EXCHANGE IN $s$ AND $\alpha$}

We here describe in detail how $F_{\mathrm{x}}(s, \alpha)$ is able to recover the GE4 for exchange [47,109] with one degree of freedom. Typically, the GE4 is written in $s^{2}$ and

$$
q=\frac{\nabla^{2} n}{4\left(3 \pi^{2}\right)^{2 / 3} n^{5 / 3}}
$$

as

$$
\begin{aligned}
F_{\mathrm{x}}^{\mathrm{GE} 4}(s, q) \sim & 1+\frac{10}{81} s^{2}+\frac{146}{2025} q^{2}-\frac{73}{405} s^{2} q \\
& +0 \cdot s^{4}+\mathcal{O}\left(\nabla^{6}\right) \text { as } s, q \rightarrow 0
\end{aligned}
$$

assuming a vanishing $s^{4}$ coefficient as the best numerical estimate. As $\alpha \rightarrow 1$ in the homogeneous electron gas limit, the GE4 for exchange in $s$ and $\alpha$ has the general form of Eq. (24) with its five coefficients $\mu_{s}, \mu_{\alpha}, C_{s}, C_{s \alpha}$, and $C_{\alpha}$ to be determined as follows: First, $\alpha=\tau / \tau^{\text {unif }}-5 s^{2} / 3$ is expanded by utilizing the GE4 of $\tau[110,111]$ to obtain

$$
\begin{aligned}
\alpha \sim 1 & -\frac{40}{27} s^{2}+\frac{20}{9} q+\frac{1}{81}\left[-14 q^{2}+\frac{140}{3} s^{2} q-48 s^{2}+12 \frac{\nabla^{2}\left(\nabla^{2} n\right)}{2^{4}\left(3 \pi^{2}\right)^{4 / 3} n^{7 / 3}}\right. \\
& \left.-30 \frac{\nabla n \cdot \nabla\left(\nabla^{2} n\right)}{2^{4}\left(3 \pi^{2}\right)^{4 / 3} n^{10 / 3}}-7 \frac{\nabla^{2}|\nabla n|^{2}}{2^{4}\left(3 \pi^{2}\right)^{4 / 3} n^{10 / 3}}+\frac{92}{3} \frac{\nabla n \cdot \nabla|\nabla n|^{2}}{2^{4}\left(3 \pi^{2}\right)^{4 / 3} n^{13 / 3}}\right]+\mathcal{O}\left(\nabla^{6}\right) .
\end{aligned}
$$

Inserting this expansion into Eq. (24) gives

$$
\begin{aligned}
F_{\mathrm{x}}^{\mathrm{GE} 4}(s, \alpha) \sim & +\left(\mu_{s}-\frac{40}{27} \mu_{\alpha}\right) s^{2}+\left(\frac{20}{9} \mu_{\alpha}\right) q+\left(-\frac{14}{81} \mu_{\alpha}+\frac{400}{81} C_{\alpha}\right) q^{2}+\left(\frac{140}{243} \mu_{\alpha}+\frac{20}{9} C_{s \alpha}-\frac{1600}{243} C_{\alpha}\right) s^{2} q \\
& +\left(-\frac{48}{81} \mu_{\alpha}+C_{s}-\frac{40}{27} C_{s \alpha}+\frac{1600}{729} C_{\alpha}\right) s^{4}+\mu_{\alpha}\left[\frac{4}{27} \frac{\nabla^{2}\left(\nabla^{2} n\right)}{2^{4}\left(3 \pi^{2}\right)^{4 / 3} n^{7 / 3}}-\frac{10}{27} \frac{\nabla n \cdot \nabla\left(\nabla^{2} n\right)}{2^{4}\left(3 \pi^{2}\right)^{4 / 3} n^{10 / 3}}\right. \\
& \left.-\frac{7}{81} \frac{\nabla^{2}|\nabla n|^{2}}{2^{4}\left(3 \pi^{2}\right)^{4 / 3} n^{10 / 3}}+\frac{92}{243} \frac{\nabla n \cdot \nabla|\nabla n|^{2}}{2^{4}\left(3 \pi^{2}\right)^{4 / 3} n^{13 / 3}}\right]+\mathcal{O}\left(\nabla^{6}\right) .
\end{aligned}
$$

Note that, under integration by parts,

$$
\int n^{4 / 3} s^{2} d^{3} r=3 \int n^{4 / 3} q d^{3} r .
$$

Therefore, and as we only consider $F_{\mathrm{x}}^{\mathrm{GE} 4}(s, \alpha)$ under the exchange energy integral $E_{\mathrm{x}}=A_{\mathrm{x}} \int n^{4 / 3} F_{\mathrm{x}}^{\mathrm{GE} 4}(s, \alpha) d^{3} r$, the separate occurrences of $q$ in Eq. (A4) can be recast to $s^{2} / 3$. Moreover, one can transform the remaining fourth-order terms to the variables $s$ and $q$ in similar fashion via integration by parts:

$$
\begin{aligned}
& \int n^{4 / 3} \frac{\nabla^{2}\left(\nabla^{2} n\right)}{2^{4}\left(3 \pi^{2}\right)^{4 / 3} n^{7 / 3}} d^{3} r=\int n^{4 / 3}\left(2 s^{2} q-q^{2}\right) d^{3} r, \\
& \int n^{4 / 3} \frac{\nabla n \cdot \nabla\left(\nabla^{2} n\right)}{2^{4}\left(3 \pi^{2}\right)^{4 / 3} n^{10 / 3}} d^{3} r=\int n^{4 / 3}\left(2 s^{2} q-q^{2}\right) d^{3} r, \\
& \int n^{4 / 3} \frac{\nabla^{2}|\nabla n|^{2}}{2^{4}\left(3 \pi^{2}\right)^{4 / 3} n^{10 / 3}} d^{3} r=\int n^{4 / 3}\left(-2 s^{2} q+6 s^{4}\right) d^{3} r, \\
& \int n^{4 / 3} \frac{\nabla n \cdot \nabla|\nabla n|^{2}}{2^{4}\left(3 \pi^{2}\right)^{4 / 3} n^{13 / 3}} d^{3} r=\int n^{4 / 3}\left(-s^{2} q+3 s^{4}\right) d^{3} r .
\end{aligned}
$$


This allows us to write Eq. (A4) as

$$
\begin{aligned}
F_{\mathrm{x}}^{\mathrm{GE} 4}(s, \alpha) \sim & +\left(\mu_{s}-\frac{20}{27} \mu_{\alpha}\right) s^{2}+\left(\frac{4}{81} \mu_{\alpha}+\frac{400}{81} C_{\alpha}\right) q^{2}+\left(-\frac{2}{27} \mu_{\alpha}+\frac{20}{9} C_{s \alpha}-\frac{1600}{243} C_{\alpha}\right) s^{2} q \\
& +\left(\frac{2}{81} \mu_{\alpha}+C_{s}-\frac{40}{27} C_{s \alpha}+\frac{1600}{729} C_{\alpha}\right) s^{4}+\mathcal{O}\left(\nabla^{6}\right) .
\end{aligned}
$$

Finally, comparison with the GE4 of Eq. (24) yields the values for the coefficients already given by Eqs. (25) and (26) with one degree of freedom, the choice of $\mu_{\alpha}$. When choosing $\mu_{\alpha}=0$ the resulting GE4 is identical to the one given in the Supplemental Material of Ref. [52].

\section{APPENDIX B: HYDROGEN ATOM NORM}

A meta-GGA for exchange defined by an enhancement factor $F_{\mathrm{x}}(\alpha)$ that only depends on $\alpha$ reduces for any singleorbital system to a multiple of LDA,

$$
\begin{aligned}
E_{\mathrm{x}}^{\mathrm{mGGA}}[n] & =A_{\mathrm{x}} \int F_{\mathrm{x}}(\alpha(\mathbf{r})) n^{4 / 3}(\mathbf{r}) d^{3} r \\
& =F_{\mathrm{x}}(0) A_{\mathrm{x}} \int n^{4 / 3}(\mathbf{r}) d^{3} r=F_{\mathrm{x}}(0) E_{\mathrm{x}}^{\mathrm{LDA}}[n]
\end{aligned}
$$

since $\alpha(\mathbf{r}) \equiv 0$ in the single-orbital limit. Given the completely spin-polarized exact hydrogen ground-state density

$$
n_{\mathrm{H}}(\mathbf{r})=\frac{1}{\pi a_{0}{ }^{3}} \exp \left(-2 r / a_{0}\right),
$$

the associated exchange energy of the meta-GGA is obtained by virtue of the spin-scaling relation for exchange [78] to be

$$
\begin{aligned}
E_{\mathrm{x}}^{\mathrm{mGGA}}\left[n_{\mathrm{H}}, 0\right] & =F_{\mathrm{x}}(0) E_{\mathrm{x}}^{\mathrm{LDA}}\left[2 n_{\mathrm{H}}\right] / 2 \\
& =-F_{\mathrm{x}}(0) \frac{81 e^{2}}{128 a_{0}}\left(\frac{3}{4 \pi^{2}}\right)^{1 / 3} .
\end{aligned}
$$

Consequently, the exchange energy of the meta-GGA matches the exact value at the hydrogen ground-state density

$$
\begin{aligned}
E_{\mathrm{x}}\left[n_{\mathrm{H}}, 0\right] & =-E_{\mathrm{H}}\left[n_{\mathrm{H}}\right] \\
& =-\frac{e^{2}}{2} \iint \frac{n_{\mathrm{H}}(\mathbf{r}) n_{\mathrm{H}}\left(\mathbf{r}^{\prime}\right)}{\left|\mathbf{r}-\mathbf{r}^{\prime}\right|} d^{3} r d^{3} r^{\prime}=-\frac{5 e^{2}}{16 a_{0}},
\end{aligned}
$$

if one chooses

$$
F_{\mathrm{X}}(0)=c_{\mathrm{H}}:=\frac{40}{81}\left(\frac{4 \pi^{2}}{3}\right)^{1 / 3} \approx 1.16588
$$

[cf. Eq. (19)].

\section{APPENDIX C: ULTRANONLOCALITY IN RECIPROCAL SPACE}

Having explicitly demonstrated that and how a meta-GGA can achieve ultranonlocality associated with the derivative discontinuity in real space and for finite systems, a comparison with previous work on ultranonlocality in reciprocal space and extended systems is worthwhile. Nazarov and Vignale [88] showed via a reciprocal space analysis that the xc kernel $f_{\mathrm{xc}}$ of a meta-GGA is capable of long-rangedness, which also is an aspect of ultranonlocality. When thinking about $f_{\mathrm{xc}}$, different aspects of ultranonlocality are associated [90,134] with a singularity of the type

$$
f_{\mathrm{xc}, \mathbf{0 0}}(\mathbf{q}) \sim \frac{\beta}{q^{2}}
$$

in the optical limit $\mathbf{q} \rightarrow 0$. Using an averaging approximation similar to the one that we use in Eq. (12) to demonstrate the relation between $\Delta_{\mathrm{x}}$ and $\overline{\partial e_{\mathrm{x}} / \partial \tau}$, they extract the singularity to be

$$
f_{\mathrm{xc}, \mathbf{G G}}(\mathbf{q}) \approx-\frac{\overline{\partial e_{\mathrm{xc}}}}{\partial \tau} \chi_{\mathrm{s}, \mathbf{G} \mathbf{G}^{\prime}}^{-1}(\mathbf{q}),
$$

where $\chi_{\mathrm{s}}^{-1}$ is the inverse of the noninteracting KS response function. Using this expression as an approximation to the full kernel, they calculate the excitation spectra of semiconductors with a special focus on the excitonic peak. These spectra and comparison with the work of Reining et al. [134] then suggest $\beta<0$, and hence $\overline{\partial e_{\mathrm{xc}} / \partial \tau}<0$ in order to capture the electron-hole interaction. Their analysis thus leads to the opposite sign of $\partial e_{\mathrm{xc}} / \partial \tau$ that we reach based on the functional derivative of Eq. (10) and the fact that $\Delta_{\mathrm{x}}$ is determined by its last term.

To resolve this seemingly contradiction, we first note that Ref. [88] focuses on a different physical problem than we do. Nazarov and Vignale [88] study the binding of excitons. As argued in Ref. [134], this merely requires a static long-range contribution to the kernel,

$$
\Delta f_{\mathrm{xc}}\left(\mathbf{r}, \mathbf{r}^{\prime}\right)=\frac{\beta}{4 \pi\left|\mathbf{r}-\mathbf{r}^{\prime}\right|}
$$

with $\beta<0$. We further note that obtaining properties of $f_{\mathrm{xc}}$ and $v_{\mathrm{xc}}$ that are long range in real space correctly with semilocal approximations is only possible within narrow conditions and typically leads to divergences of the $\mathrm{KS}$ potential for many finite systems [71,72].

In our work here, we focus on the ultranonlocality that is associated with potential step structures and the derivative discontinuity. Our tests were done for finite systems, but Ghosez et al. [90] demonstrated that this type of ultranonlocality is also present in solids. When calculating the polarization dependence of solids, which is in close analogy to the ultranonlocality effects in the electrical response of molecular chains, this ultranonlocality is connected to a positive $(\beta>0)$ singularity of the form of Eq. (C1) (see, e.g., Fig. 1 of Ref. [90]). Within the approximation of Eq. (C2) this singularity can in turn be associated with $\overline{\partial e_{\mathrm{xc}} / \partial \tau}>0$. Therefore, the analysis of Ghosez et al. [90] is in line with our arguments.

Finally, we note that also Ref. [134] finds a positive contribution to the singularity in $f_{\mathrm{xc}}$ that stems from the energy shift between KS and quasiparticle eigenvalues. Within KS 
TABLE IV. Dimensions of ellipsoidal boundaries used for the hydrogen chain polarizability calculation in PARSEC in $a_{0}$.

\begin{tabular}{lccccc}
\hline \hline Semiaxis & $\mathrm{H}_{4}$ & $\mathrm{H}_{6}$ & $\mathrm{H}_{8}$ & $\mathrm{H}_{12}$ & $\mathrm{H}_{18}$ \\
\hline Parallel & 15.0 & 17.5 & 20.0 & 25.0 & 32.5 \\
Perp. & 10.0 & 10.0 & 10.0 & 10.0 & 13.0 \\
\hline
\end{tabular}

theory this energy shift is accounted for by the derivative discontinuity $\Delta_{\mathrm{xc}}$. In the calculations of Ref. [134], however, it is absorbed into an energy shift of the starting $\chi_{s}$.

\section{APPENDIX D: HYDROGEN CHAIN POLARIZABILITY CALCULATION DETAILS}

The polarizabilities of hydrogen chains were calculated with a locally modified version of the publicly available electronic structure program PARSEC [95] on a real-space grid with a grid spacing of $0.27 a_{0}$. The linear chains with alternating $\mathrm{H}-\mathrm{H}$ distances of $2 a_{0}$ and $3 a_{0}$ are centered in an ellipsoid with dimensions as listed in Table IV. All calculations were performed self-consistently with a functional independent pseudopotential [135], an energy convergence criterion for the KS iterations of $5 \times 10^{-7} E_{\mathrm{h}}$, and a field strength of $5 \times 10^{-5} E_{\mathrm{h}} / a_{0}$. To obtain the KS potential based on the OEP scheme via $S$-Iterations [81], $S$ was converged to at least $3 \times 10^{-7} E_{\mathrm{h}}$. The implementation of all meta-GGAs with the exception of PoC, TASK, and SCAN is built on an interface to LIBXC version 4.3.4 [136]. We found that for several of the meta-GGAs that are available in LIBXC [136], e.g., TPSS [47], revTPSS [50], TM [55], and the Minnesota meta-GGAs [49], the grid-based calculations are difficult to converge as their potentials show rapidly varying features. This type of observation is in line with the arguments of, e.g., Ref. [114]. Therefore, the results reported here are restricted to numerically benevolent meta-GGAs. For the calculations with the MVS functional we found that the OEP calculations converged very slowly for the longer hydrogen chains.
Therefore, for the MVS functional [54], Table I reports OEP polarizabilities for $\mathrm{H}_{4}$ and $\mathrm{H}_{6}$, but the values reported for $\mathrm{H}_{8}$, $\mathrm{H}_{12}$, and $\mathrm{H}_{18}$ are based on the KLI approximation [82]. All other values are OEP based.

\section{APPENDIX E: $\mathrm{H}_{8}-\mathrm{H}_{8}$ CHARGE-TRANSFER CALCULATION DETAILS}

All calculations for the $\mathrm{H}_{8}-\mathrm{H}_{8}$ charge-transfer system were performed spin polarized, self-consistently on a realspace grid with BTDFT [40] using Troullier-Martins LDA pseudopotentials [137], ellipsoidal boundaries with semiaxis of $20 a_{0}$ perpendicular to and $32 a_{0}$ along the chains, and a grid spacing of $0.3 a_{0}$. For the KS iterations, an energy convergence criterion of $5 \times 10^{-6} E_{\mathrm{h}}$ was used. The two hydrogen chains, each containing eight hydrogen atoms separated by $1 \AA$, are centered in the ellipsoid with a mutual distance of $8 \AA$. Due to the nature of the situation with nearly degenerate ground-state solutions, the calculations are numerically very challenging (cf. Refs. [9,105]). The LDA calculations were performed with occupation numbers following from a Fermi-Dirac distribution with a temperature of $800 \mathrm{~K}$, as zero-temperature calculations cannot be converged once the external field strength leads to (a fractional) transfer of charge. The meta-GGA calculations in the KLI approximation [82] were performed at $0 \mathrm{~K}$. For the PoC meta-GGA, convergence was possible for any considered field strength. We can carefully converge two nearly degenerate ground states with an integer charge of 8 and 9 electrons, respectively, even for field strengths below and above the critical field strength of $3.7 \times 10^{9} \mathrm{~V} / \mathrm{m}$, at which an integer electron transfer occurs. Consequently, the associated Fig. 3 only shows the solution of lower total energy at each field strength and the critical field strength is given by the energy crossing point of both solutions. While the TASK functional does not allow for a numerical stable solution from field strengths of $3.0 \times 10^{9} \mathrm{~V} / \mathrm{m}$ to $5.8 \times 10^{9} \mathrm{~V} / \mathrm{m}$, it still improves considerably over LDA as it allows to counteract the charge transfer up to $2.8 \times 10^{9} \mathrm{~V} / \mathrm{m}$ and yields a broad integer electron plateau starting at $6.0 \times 10^{9} \mathrm{~V} / \mathrm{m}$.
[1] J. P. Perdew and Y. Wang, Accurate and simple density functional for the electronic exchange energy: Generalized gradient approximation, Phys. Rev. B 33, 8800 (1986).

[2] A. D. Becke, Density-functional exchange-energy approximation with correct asymptotic behavior, Phys. Rev. A 38, 3098 (1988).

[3] C. Lee, W. Yang, and R. G. Parr, Development of the ColleSalvetti correlation-energy formula into a functional of the electron density, Phys. Rev. B 37, 785 (1988).

[4] J. P. Perdew, K. Burke, and M. Ernzerhof, Generalized Gradient Approximation Made Simple, Phys. Rev. Lett. 77, 3865 (1996).

[5] R. Armiento and A. E. Mattsson, Functional designed to include surface effects in self-consistent density functional theory, Phys. Rev. B 72, 085108 (2005).

[6] A. D. Becke, Density-functional thermochemistry. III. The role of exact exchange, J. Chem. Phys. 98, 5648 (1993).
[7] J. P. Perdew and M. Levy, Physical Content of the Exact Kohn-Sham Orbital Energies: Band Gaps and Derivative Discontinuities, Phys. Rev. Lett. 51, 1884 (1983).

[8] R. W. Godby, M. Schlüter, and L. J. Sham, Accurate Exchange-Correlation Potential for Silicon and Its Discontinuity on Addition of an Electron, Phys. Rev. Lett. 56, 2415 (1986).

[9] S.-H. Ke, H. U. Baranger, and W. Yang, Role of the exchangecorrelation potential in ab initio electron transport calculations, J. Chem. Phys. 126, 201102 (2007).

[10] A. J. Cohen, P. Mori-Sánchez, and W. Yang, Challenges for density functional theory, Chem. Rev. 112, 289 (2012).

[11] G. Kresse, A. Gil, and P. Sautet, Significance of single-electron energies for the description of CO on Pt(111), Phys. Rev. B 68, 073401 (2003).

[12] S. Luo, Y. Zhao, and D. G. Truhlar, Improved CO adsorption energies, site preferences, and surface formation energies 
from a meta-generalized gradient approximation exchangecorrelation functional, M06-L, J. Phys. Chem. Lett. 3, 2975 (2012).

[13] S. J. A. van Gisbergen, P. R. T. Schipper, O. V. Gritsenko, E. J. Baerends, J. G. Snijders, B. Champagne, and B. Kirtman, Electric Field Dependence of the Exchange-Correlation Potential in Molecular Chains, Phys. Rev. Lett. 83, 694 (1999).

[14] P. Mori-Sánchez, Q. Wu, and W. Yang, Accurate polymer polarizabilities with exact exchange density-functional theory, J. Chem. Phys. 119, 11001 (2003).

[15] S. Kümmel, L. Kronik, and J. P. Perdew, Electrical Response of Molecular Chains from Density Functional Theory, Phys. Rev. Lett. 93, 213002 (2004).

[16] J. P. Perdew, R. G. Parr, M. Levy, and J. L. Balduz, Jr., Density-Functional Theory for Fractional Particle Number: Derivative Discontinuities of the Energy, Phys. Rev. Lett. 49, 1691 (1982).

[17] D. Tozer, Relationship between long-range charge-transfer excitation energy error and integer discontinuity in Kohn-Sham theory, J. Chem. Phys. 119, 12697 (2003).

[18] M. Mundt and S. Kümmel, Derivative Discontinuities in TimeDependent Density-Functional Theory, Phys. Rev. Lett. 95, 203004 (2005).

[19] M. van Faassen and N. Maitra, Improved exchange-correlation potential for polarizability and dissociation in density functional theory, J. Chem. Phys. 126, 191106 (2007).

[20] P. Elliott, J. I. Fuks, A. Rubio, and N. T. Maitra, Universal Dynamical Steps in the Exact Time-Dependent ExchangeCorrelation Potential, Phys. Rev. Lett. 109, 266404 (2012).

[21] D. Hofmann and S. Kümmel, Integer particle preference during charge transfer in Kohn-Sham theory, Phys. Rev. B 86, 201109(R) (2012).

[22] M. J. P. Hodgson, E. Kraisler, A. Schild, and E. K. U. Gross, How interatomic steps in the exact Kohn-Sham potential relate to derivative discontinuities of the energy, Phys. Chem. Lett. 8, 5974 (2017).

[23] A. J. Cohen, P. Mori-Sánchez, and W. Yang, Insights into current limitations of density functional theory, Science 321, 792 (2008).

[24] P. Mori-Sánchez, A. J. Cohen, and W. Yang, Localization and Delocalization Errors in Density Functional Theory and Implications for Band-Gap Prediction, Phys. Rev. Lett. 100, 146401 (2008).

[25] B. G. Janesko, Reducing density-driven error without exact exchange, Phys. Chem. Chem. Phys. 19, 4793 (2017).

[26] A. Wasserman, J. Nafziger, K. Jiang, M.-C. Kim, E. Sim, and K. Burke, The importance of being inconsistent, Annu. Rev. Phys. Chem. 68, 555 (2017).

[27] J. P. Perdew and A. Zunger, Self-interaction correction to density-functional approximations for many-electron systems, Phys. Rev. B 23, 5048 (1981).

[28] W. M. Temmerman, H. Winter, Z. Szotek, and A. Svane, Cu Valency Change Induced by O Doping in YBCO, Phys. Rev. Lett. 86, 2435 (2001).

[29] O. A. Vydrov, G. E. Scuseria, and J. P. Perdew, Tests of functionals for systems with fractional electron number, J. Chem. Phys. 126, 154109 (2007).

[30] T. Körzdörfer, M. Mundt, and S. Kümmel, Electrical Response of Molecular Systems: The Power of Self-Interaction
Corrected Kohn-Sham Theory, Phys. Rev. Lett. 100, 133004 (2008).

[31] G. Borghi, A. Ferretti, N. L. Nguyen, I. Dabo, and N. Marzari, Koopmans-compliant functionals and their performance against reference molecular data, Phys. Rev. B 90, 075135 (2014).

[32] J. Erhard, P. Bleiziffer, and A. Görling, Power Series Approximation for the Correlation Kernel Leading to Kohn-Sham Methods Combining Accuracy, Computational Efficiency, and General Applicability, Phys. Rev. Lett. 117, 143002 (2016).

[33] J. Jornet-Somoza, J. Alberdi-Rodriguez, B. F. Milne, X. Andrade, M. A. L. Marques, F. Nogueira, M. J. T. Oliveira, J. J. P. Stewart, and A. Rubio, Insights into color-tuning of chlorophyll optical response in green plants, Phys. Chem. Chem. Phys. 17, 26599 (2015).

[34] C. Curutchet and B. Mennucci, Quantum chemical studies of light harvesting, Chem. Rev. 117, 294 (2017).

[35] I. Schelter, J. M. Foerster, A. T. Gardiner, A. W. Roszak, R. J. Cogdell, G. M. Ullmann, T. B. de Queiroz, and S. Kümmel, Assessing density functional theory in real-time and real-space as a tool for studying bacteriochlorophylls and the light-harvesting complex 2, J. Chem. Phys. 151, 134114 (2019).

[36] L. Cupellini, S. Jurinovich, M. Campetella, S. Caprasecca, C. A. Guido, S. M. Kelly, A. T. Gardiner, R. Cogdell, and B. Mennucci, An ab initio description of the excitonic properties of LH2 and their temperature dependence, J. Phys. Chem. B 120, 11348 (2016).

[37] A. Anda, L. De Vico, and T. Hansen, Intermolecular modes between $\mathrm{LH} 2$ bacteriochlorophylls and protein residues: The effect on the excitation energies, J. Phys. Chem. B 121, 5499 (2017).

[38] D. Sundholm, A density-functional-theory study of bacteriochlorophyll b, Phys. Chem. Chem. Phys. 5, 4265 (2003).

[39] A. Dreuw and M. Head-Gordon, Failure of time-dependent density functional theory for long-range charge-transfer excited states: The zincbacteriochlorin-bacteriochlorin and bacteriochlorophyll-spheroidene complexes, J. Am. Chem. Soc. 126, 4007 (2004).

[40] I. Schelter and S. Kümmel, Accurate evaluation of realtime density functional theory providing access to challenging electron dynamics, J. Chem. Theory Comput. 14, 1910 (2018).

[41] A. D. Becke, Local exchange-correlation approximations and first-row molecular dissociation energies, Int. J. Quantum Chem. 27, 585 (1985).

[42] A. D. Becke, Density-functional thermochemistry. IV. A new dynamical correlation functional and implications for exact-exchange mixing, J. Chem. Phys. 104, 1040 (1996).

[43] A. D. Becke, A new inhomogeneity parameter in densityfunctional theory, J. Chem. Phys. 109, 2092 (1998).

[44] T. V. Voorhis and G. E. Scuseria, A novel form for the exchange-correlation energy functional, J. Chem. Phys. 109, 400 (1998).

[45] J. P. Perdew, S. Kurth, A. Zupan, and P. Blaha, Accurate Density Functional with Correct Formal Properties: A Step Beyond the Generalized Gradient Approximation, Phys. Rev. Lett. 82, 2544 (1999). 
[46] A. D. Boese and N. C. Handy, New exchange-correlation density functionals: The role of the kinetic-energy density, J. Chem. Phys. 116, 9559 (2002).

[47] J. Tao, J. P. Perdew, V. N. Staroverov, and G. E. Scuseria, Climbing the Density Functional Ladder: Nonempirical MetaGeneralized Gradient Approximation Designed for Molecules and Solids, Phys. Rev. Lett. 91, 146401 (2003).

[48] K. Pernal, R. Podeszwa, K. Patkowski, and K. Szalewicz, Dispersionless Density Functional Theory, Phys. Rev. Lett. 103, 263201 (2009).

[49] Y. Zhao and D. G. Truhlar, A new local density functional for main-group thermochemistry, transition metal bonding, thermochemical kinetics, and noncovalent interactions, J. Chem. Phys. 125, 194101 (2006); R. Peverati and D. G. Truhlar, An improved and broadly accurate local approximation to the exchange-correlation density functional: The MN12-L functional for electronic structure calculations in chemistry and physics, Phys. Chem. Chem. Phys. 14, 13171 (2012); H. S. Yu, X. He, and D. G. Truhlar, MN15-L: A new local exchange-correlation functional for Kohn-Sham density functional theory with broad accuracy for atoms, molecules, and solids, J. Chem. Theory Comput. 12, 1280 (2016).

[50] J. P. Perdew, A. Ruzsinszky, G. I. Csonka, L. A. Constantin, and J. Sun, Workhorse Semilocal Density Functional for Condensed Matter Physics and Quantum Chemistry, Phys. Rev. Lett. 103, 026403 (2009).

[51] J. Sun, B. Xiao, Y. Fang, R. Haunschild, P. Hao, A. Ruzsinszky, G. I. Csonka, G. E. Scuseria, and J. P. Perdew, Density Functionals that Recognize Covalent, Metallic, and Weak Bonds, Phys. Rev. Lett. 111, 106401 (2013).

[52] J. Sun, A. Ruzsinszky, and J. P. Perdew, Strongly Constrained and Appropriately Normed Semilocal Density Functional, Phys. Rev. Lett. 115, 036402 (2015).

[53] J. Sun, B. Xiao, and A. Ruzsinszky, Communication: Effect of the orbital-overlap dependence in the meta generalized gradient approximation, J. Chem. Phys. 137, 051101 (2012); J. Sun, R. Haunschild, B. Xiao, I. W. Bulik, G. E. Scuseria, and J. P. Perdew, Semilocal and hybrid meta-generalized gradient approximations based on the understanding of the kineticenergy-density dependence, ibid. 138, 044113 (2013).

[54] J. Sun, J. P. Perdew, and A. Ruzsinszky, Semilocal density functional obeying a strongly tightened bound for exchange, Proc. Natl. Acad. Sci. USA 112, 685 (2015).

[55] J. Tao and Y. Mo, Accurate Semilocal Density Functional for Condensed-Matter Physics and Quantum Chemistry, Phys. Rev. Lett. 117, 073001 (2016).

[56] F. Della Sala, E. Fabiano, and L. A. Constantin, Kineticenergy-density dependent semilocal exchange-correlation functionals, Int. J. Quantum Chem. 116, 1641 (2016).

[57] M. G. Medvedev, I. S. Bushmarinov, J. Sun, J. P. Perdew, and K. A. Lyssenko, Density functional theory is straying from the path toward the exact functional, Science 355, 49 (2017).

[58] T. Schmidt, E. Kraisler, A. Makmal, L. Kronik, and S. Kümmel, A self-interaction-free local hybrid functional: Accurate binding energies vis-à-vis accurate ionization potentials from Kohn-Sham eigenvalues, J. Chem. Phys. 140, 18A510 (2014).

[59] V. Polo, E. Kraka, and D. Cremer, Electron correlation and the self-interaction error of density functional theory, Mol. Phys. 100, 1771 (2002).
[60] P. Verma and R. J. Bartlett, Increasing the applicability of density functional theory. III. Do consistent Kohn-Sham density functional methods exist? J. Chem. Phys. 137, 134102 (2012).

[61] E. Trushin, M. Betzinger, S. Blügel, and A. Görling, Band gaps, ionization potentials, and electron affinities of periodic electron systems via the adiabatic-connection fluctuationdissipation theorem, Phys. Rev. B 94, 075123 (2016).

[62] A. D. Becke and E. R. Johnson, A simple effective potential for exchange, J. Chem. Phys. 124, 221101 (2006).

[63] R. Armiento, S. Kümmel, and T. Körzdörfer, Electrical response of molecular chains in density functional theory: Ultranonlocal response from a semilocal functional, Phys. Rev. B 77, 165106 (2008).

[64] F. Tran and P. Blaha, Accurate Band Gaps of Semiconductors and Insulators with a Semilocal Exchange-Correlation Potential, Phys. Rev. Lett. 102, 226401 (2009).

[65] E. Räsänen, S. Pittalis, and C. R. Proetto, Universal correction for the Becke-Johnson exchange potential, J. Chem. Phys. 132, 044112 (2010).

[66] F. Tran, P. Blaha, M. Betzinger, and S. Blügel, Comparison between exact and semilocal exchange potentials: An allelectron study for solids, Phys. Rev. B 91, 165121 (2015).

[67] A. P. Gaiduk, S. K. Chulkov, and V. N. Staroverov, Reconstruction of density functionals from Kohn-Sham potentials by integration along density scaling paths, J. Chem. Theory Comput. 5, 699 (2009).

[68] A. Karolewski, R. Armiento, and S. Kümmel, Polarizabilities of polyacetylene from a field-counteracting semilocal functional, J. Chem. Theory Comput. 5, 712 (2009).

[69] A. Karolewski, R. Armiento, and S. Kümmel, Electronic excitations and the Becke-Johnson potential: The need for and the problem of transforming model potentials to functional derivatives, Phys. Rev. A 88, 052519 (2013).

[70] R. Armiento and S. Kümmel, Orbital Localization, Charge Transfer, and Band Gaps in Semilocal Density-Functional Theory, Phys. Rev. Lett. 111, 036402 (2013).

[71] T. Aschebrock, R. Armiento, and S. Kümmel, Orbital nodal surfaces: Topological challenges for density functionals, Phys. Rev. B 95, 245118 (2017).

[72] T. Aschebrock, R. Armiento, and S. Kümmel, Challenges for semilocal density functionals with asymptotically nonvanishing potentials, Phys. Rev. B 96, 075140 (2017).

[73] J. Garhammer, F. Hofmann, R. Armiento, and S. Kümmel, On the challenge to improve the density response with unusual gradient approximations, Eur. Phys. J. B 91, 159 (2018).

[74] F. Tran, J. Doumont, L. Kalantari, A. W. Huran, M. A. L. Marques, and P. Blaha, Semilocal exchange-correlation potentials for solid-state calculations: Current status and future directions, J. Appl. Phys. 126, 110902 (2019).

[75] M. Levy and J. P. Perdew, Hellmann-Feynman, virial, and scaling requisites for the exact universal density functionals. Shape of the correlation potential and diamagnetic susceptibility for atoms, Phys. Rev. A 32, 2010 (1985).

[76] A. Ruzsinszky, J. Sun, B. Xiao, and G. I. Csonka, A metaGGA made free of the order of limits anomaly, J. Chem. Theory Comput. 8, 2078 (2012).

[77] F. Della Sala, E. Fabiano, and L. A. Constantin, KohnSham kinetic energy density in the nuclear and asymptotic regions: Deviations from the von Weizsäcker behavior and 
applications to density functionals, Phys. Rev. B 91, 035126 (2015).

[78] G. L. Oliver and J. P. Perdew, Spin-density gradient expansion for the kinetic energy, Phys. Rev. A 20, 397 (1979).

[79] O. V. Gritsenko, R. van Leeuwen, E. van Lenthe, and E. J. Baerends, Self-consistent approximation to the Kohn-Sham exchange potential, Phys. Rev. A 51, 1944 (1995).

[80] T. Grabo, T. Kreibich, S. Kurth, and E. K. U. Gross, in Strong Coulomb Correlation in Electronic Structure: Beyond the Local Density Approximation, edited by V. Anisimov (Gordon \& Breach, Tokyo, 2000), pp. 203-311.

[81] S. Kümmel and J. P. Perdew, Simple Iterative Construction of the Optimized Effective Potential for Orbital Functionals, Including Exact Exchange, Phys. Rev. Lett. 90, 043004 (2003).

[82] J. B. Krieger, Y. Li, and G. J. Iafrate, Construction and application of an accurate local spin-polarized Kohn-Sham potential with integer discontinuity: Exchange-only theory, Phys. Rev. A 45, 101 (1992).

[83] M. Grüning, O. V. Gritsenko, and E. J. Baerends, Exchange potential from the common energy denominator approximation for the Kohn-Sham Green's function: Application to (hyper)polarizabilities of molecular chains, J. Chem. Phys. 116, 6435 (2002).

[84] S. Kümmel and L. Kronik, Orbital-dependent density functionals: Theory and applications, Rev. Mod. Phys. 80, 3 (2008).

[85] F. G. Eich and M. Hellgren, Derivative discontinuity and exchange-correlation potential of meta-GGAs in densityfunctional theory, J. Chem. Phys. 141, 224107 (2014).

[86] G. K.-L. Chan, A fresh look at ensembles: Derivative discontinuities in density functional theory, J. Chem. Phys. 110, 4710 (1999).

[87] E. Sagvolden and J. P. Perdew, Discontinuity of the exchangecorrelation potential: Support for assumptions used to find it, Phys. Rev. A 77, 012517 (2008).

[88] V. U. Nazarov and G. Vignale, Optics of Semiconductors from Meta-Generalized-Gradient-Approximation-Based Time-Dependent Density-Functional Theory, Phys. Rev. Lett. 107, 216402 (2011).

[89] J. P. Perdew, A. Ruzsinszky, J. Sun, and K. Burke, Gedanken densities and exact constraints in density functional theory, J. Chem. Phys. 140, 18A533 (2014).

[90] P. Ghosez, X. Gonze, and R. W. Godby, Long-wavelength behavior of the exchange-correlation kernel in the Kohn-Sham theory of periodic systems, Phys. Rev. B 56, 12811 (1997).

[91] A. D. Becke and K. E. Edgecombe, A simple measure of electron localization in atomic and molecular systems, J. Chem. Phys. 92, 5397 (1990).

[92] A. Savin, O. Jepsen, J. Flad, O. K. Andersen, H. Preuss, and H. G. von Schnering, Electron localization in solid-state structures of the elements: the diamond structure, Angew. Chem., Int. Ed. Engl. 31, 187 (1992); B. Silvi and A. Savin, Classification of chemical bonds based on topological, Nature (London) 371, 683 (1994); A. Savin, R. Nesper, S. Wengert, and T. F. Fässler, ELF: The electron localization function, Angew. Chem., Int. Ed. Engl. 36, 1808 (1997).

[93] J. P. Perdew and Y. Wang, Accurate and simple analytic representation of the electron-gas correlation energy, Phys. Rev. B 45, 13244 (1992).
[94] B. Champagne, D. H. Mosley, M. Vračko, and J.-M. André, Electron-correlation effects on the static longitudinal polarizability of polymeric chains, Phys. Rev. A 52, 178 (1995).

[95] L. Kronik, A. Makmal, M. L. Tiago, M. M. G. Alemany, M. Jain, X. Huang, Y. Saad, and J. R. Chelikowsky, PARSEC the pseudopotential algorithm for real-space electronic structure calculations: recent advances and novel applications to nano-structures, Phys. Status Solidi B 243, 1063 (2006).

[96] M. Mundt, S. Kümmel, B. Huber, and M. Moseler, Photoelectron spectra of sodium clusters: The problem of interpreting Kohn-Sham eigenvalues, Phys. Rev. B 73, 205407 (2006).

[97] M. van Faassen, P. L. de Boeij, R. van Leeuwen, J. A. Berger, and J. G. Snijders, Ultranonlocality in Time-Dependent Current-Density-Functional Theory: Application to Conjugated Polymers, Phys. Rev. Lett. 88, 186401 (2002).

[98] P. Umari, A. J. Willamson, G. Galli, and N. Marzari, Dielectric Response of Periodic Systems from Quantum Monte Carlo Calculations, Phys. Rev. Lett. 95, 207602 (2005).

[99] H. Sekino, Y. Maeda, M. Kamiya, and K. Hirao, Polarizability and second hyperpolarizability evaluation of long molecules by the density functional theory with long-range correction, J. Chem. Phys. 126, 014107 (2007).

[100] A. Ruzsinszky, J. P. Perdew, G. I. Csonka, G. E. Scuseria, and O. A. Vydrov, Understanding and correcting the selfinteraction error in the electrical response of hydrogen chains, Phys. Rev. A 77, 060502(R) (2008).

[101] A. Ruzsinszky, J. P. Perdew, and G. I. Csonka, Simple chargetransfer model to explain the electrical response of hydrogen chains, Phys. Rev. A 78, 022513 (2008).

[102] C. D. Pemmaraju, S. Sanvito, and K. Burke, Polarizability of molecular chains: A self-interaction correction approach, Phys. Rev. B 77, 121204(R) (2008).

[103] B. Champagne and B. Kirtman, Polarizabilities and second hyperpolarizabilities of hydrogen chains using the spincomponent-scaled Møller-Plesset second-order method, Int. J. Quantum Chem. 109, 3103 (2009).

[104] E. H. Lieb and S. Oxford, Improved lower bound on the indirect Coulomb energy, Int. J. Quantum Chem. 19, 427 (1981).

[105] T. Schmidt and S. Kümmel, One- and many-electron selfinteraction error in local and global hybrid functionals, Phys. Rev. B 93, 165120 (2016).

[106] J. P. Perdew, Size-consistency, self-interaction correction, and derivative discontinuity in density functional theory, Adv. Quantum Chem. 21, 113 (1990).

[107] M. Levy, Density-functional exchange correlation through coordinate scaling in adiabatic connection and correlation hole, Phys. Rev. A 43, 4637 (1991).

[108] L. Pollack and J. P. Perdew, Evaluating density functional performance for the quasi-two-dimensional electron gas, J. Phys.: Condens. Matter 12, 1239 (2000).

[109] P. S. Svendsen and U. von Barth, Gradient expansion of the exchange energy from second-order density response theory, Phys. Rev. B 54, 17402 (1996).

[110] M. Brack, B. Jennings, and Y. Chu, On the extended ThomasFermi approximation to the kinetic energy density, Phys. Lett. B 65, 1 (1976).

[111] J. P. Perdew, V. Sahni, M. K. Harbola, and R. K. Pathak, Fourth-order gradient expansion of the fermion kinetic energy: 
Extra terms for nonanalytic densities, Phys. Rev. B 34, 686 (1986).

[112] J. P. Boyd, Orthogonal rational functions on a semi-infinite interval, J. Comput. Phys. 70, 63 (1987).

[113] W. H. Press, S. A. Teukolsky, W. T. Vetterling, and B. P. Flannery, Numerical Recipes (Cambridge University Press, Cambridge, New York, 1992).

[114] J. W. Furness and J. Sun, Enhancing the efficiency of density functionals with an improved iso-orbital indicator, Phys. Rev. B 99, 041119(R) (2019).

[115] A. Makmal, S. Kümmel, and L. Kronik, Fully numerical allelectron solutions of the optimized effective potential equation for diatomic molecules, J. Chem. Theory Comput. 5, 1731 (2009).

[116] CRC Handbook of Chemistry and Physics, 98th ed., edited by J. Rumble (CRC Press, Boca Raton, FL, 2017).

[117] T. Aschebrock and S. Kümmel, Exploring local range separation: The role of spin scaling and one-electron self-interaction, J. Chem. Phys. 151, 154108 (2019).

[118] A. Kaiser and S. Kümmel, Revealing the field-counteracting term in the exact Kohn-Sham correlation potential, Phys. Rev. A 98, 052505 (2018).

[119] V. N. Staroverov, G. E. Scuseria, J. Tao, and J. P. Perdew, Tests of a ladder of density functionals for bulk solids and surfaces, Phys. Rev. B 69, 075102 (2004).

[120] J. Heyd, J. E. Peralta, G. E. Scuseria, and R. L. Martin, Energy band gaps and lattice parameters evaluated with the HeydScuseria-Ernzerhof screened hybrid functional, J. Chem. Phys. 123, 174101 (2005).

[121] R. J. Guerrero-Moreno and N. Takeuchi, First principles calculations of the ground-state properties and structural phase transformation in CdO, Phys. Rev. B 66, 205205 (2002).

[122] F. P. Koffyberg, Thermoreflectance spectra of CdO: Band gaps and band-population effects, Phys. Rev. B 13, 4470 (1976).

[123] F. Tran, P. Blaha, and K. Schwarz, Band gap calculations with Becke-Johnson exchange potential, J. Phys.: Condens. Matter 19, 196208 (2007).

[124] Z. H. Yang, H. Peng, J. Sun, and J. P. Perdew, More realistic band gaps from meta-generalized gradient approximations: Only in a generalized Kohn-Sham scheme, Phys. Rev. B 93, 205205 (2016).

[125] N. Sai, P. F. Barbara, and K. Leung, Hole Localization in Molecular Crystals from Hybrid Density Functional Theory, Phys. Rev. Lett. 106, 226403 (2011).

[126] C. Adamo and V. Barone, Toward reliable density functional methods without adjustable parameters: The PBE0 model, J. Chem. Phys. 110, 6158 (1999).
[127] A. Seidl, A. Görling, P. Vogl, J. A. Majewski, and M. Levy, Generalized Kohn-Sham schemes and the band-gap problem, Phys. Rev. B 53, 3764 (1996).

[128] J. P. Perdew, W. Yang, K. Burke, Z. Yang, E. K. U. Gross, M. Scheffler, G. E. Scuseria, T. M. Henderson, I. Y. Zhang, A. Ruzsinszky, H. Peng, J. Sun, E. Trushin, and A. Görling, Understanding band gaps of solids in generalized Kohn-Sham theory, Proc. Natl. Acad. Sci. USA 114, 2801 (2017).

[129] L. Kronik, T. Stein, S. Refaely-Abramson, and R. Baer, Excitation gaps of finite-sized systems from optimally tuned range-separated hybrid functionals, J. Chem. Theory Comput. 8, 1515 (2012).

[130] G. te Velde and E. J. Baerends, Precise density-functional method for periodic structures, Phys. Rev. B 44, 7888 (1991); G. Wiesenekker and E. J. Baerends, Quadratic integration over the three-dimensional Brillouin zone, J. Phys.: Condens. Matter 3, 6721 (1991); M. Franchini, P. H. T. Philipsen, and L. Visscher, The Becke fuzzy cells integration scheme in the Amsterdam density functional program suite, J. Comput. Chem. 34, 1819 (2013); M. Franchini, P. H. T. Philipsen, E. van Lenthe, and L. Visscher, Accurate Coulomb potentials for periodic and molecular systems through density fitting, J. Chem. Theory Comput. 10, 1994 (2014); BAND2017, SCM, Theoretical Chemistry, Vrije Universiteit, Amsterdam, The Netherlands, https://www.scm.com.

[131] H. J. Monkhorst and J. D. Pack, Special points for Brillouinzone integrations, Phys. Rev. B 13, 5188 (1976).

[132] E. Van Lenthe and E. J. Baerends, Optimized Slater-type basis sets for the elements 1-118, J. Comput. Chem. 24, 1142 (2003).

[133] P. H. T. Philipsen, E. van Lenthe, J. G. Snijders, and E. J. Baerends, Relativistic calculations on the adsorption of $\mathrm{CO}$ on the (111) surfaces of $\mathrm{Ni}, \mathrm{Pd}$, and Pt within the zeroth-order regular approximation, Phys. Rev. B 56, 13556 (1997).

[134] L. Reining, V. Olevano, A. Rubio, and G. Onida, Excitonic Effects in Solids Described by Time-Dependent Density-Functional Theory, Phys. Rev. Lett. 88, 066404 (2002)

[135] F. Gygi, Electronic-structure calculations in adaptive coordinates, Phys. Rev. B 48, 11692 (1993).

[136] S. Lehtola, C. Steigemann, M. J. Oliveira, and M. A. Marques, Recent developments in libxc - A comprehensive library of functionals for density functional theory, SoftwareX 7, 1 (2018).

[137] N. Troullier and J. L. Martins, Efficient pseudopotentials for plane-wave calculations, Phys. Rev. B 43, 1993 (1991). 